\title{
MANIFOLD-THEORETIC COMPACTIFICATIONS OF CONFIGURATION SPACES
}

\author{
DEV P. SINHA
}

\begin{abstract}
We present new definitions for and give a comprehensive treatment of the canonical compactification of configuration spaces due to Fulton-MacPherson and Axelrod-Singer in the setting of smooth manifolds, as well as a simplicial variant of this compactification. Our constructions are elementary and give simple global coordinates for the compactified configuration space of a general manifold embedded in Euclidean space. We stratify the canonical compactification, identifying the diffeomorphism types of the strata in terms of spaces of configurations in the tangent bundle, and give completely explicit local coordinates around the strata as needed to define a manifold with corners. We analyze the quotient map from the canonical to the simplicial compactification, showing it is a homotopy equivalence. We define projection maps and diagonal maps, which for the simplicial variant satisfy cosimplicial identities.
\end{abstract}

\section{Contents}

\begin{tabular}{|c|}
\hline 1. Introduction \\
\hline 1.1. The basic definitions \\
\hline 1.2. Review of previous work \\
\hline 1.3. A comment on notation. and a little lemma \\
\hline 1.4. Acknowledgements \\
\hline 2. A categorv of trees and related categories \\
\hline 3. The stratification of the basic compactificatio \\
\hline 3.1. Stratification of $C_{n}[M]$ using coordinates in \\
\hline 3.2. Statement of the main theorem \\
\hline 3.3. The auxilliarv construction. $C_{n}\left\{\mathbb{R}^{k}\right\}$ \\
\hline 3.4. Proof of Theorem 3.8 for $M=\mathbb{R}^{k}$ \\
\hline 3.5. Proof of Theorem 3.8 for general $M$ \\
\hline 4. First properties \\
\hline 4.1. Characterization in $A_{n}[M]$ and standard $\mathrm{pr}$ \\
\hline 4.2. Manifold structure, codimensions of strata. \\
\hline 4.3. The closures of strata \\
\hline 4.4. Configurations in the line and associahedra \\
\hline 5. The simplicial compactification \\
\hline 6. Diagonal and proiection maps \\
\hline References \\
\hline
\end{tabular}

\section{INTRODUCTION}

Configuration spaces are fundamental objects of study in geometry and topology, and over the past ten years, functorial compactifications of configuration spaces have been an important technical tool. We review the state of this active area after giving our definitions.

1991 Mathematics Subject Classification. Primary: 55T99. 
1.1. The basic definitions. We deal with products of spaces extensively, so we first set down some efficient notation to manage products.

Notation. If $S$ is a finite set, $X^{S}$ is the product $X^{\# S}$ where \#S is the cardinality of $S$. Consistent with this, if $\left\{X_{s}\right\}$ is a collection of spaces indexed by $S$, we let $\left(X_{s}\right)^{S}=\prod_{s \in S} X_{s}$. For coordinates in either case we use $\left(x_{s}\right)_{s \in S}$ or just $\left(x_{s}\right)$ when $S$ is understood. Similarly, a product of maps $\prod_{s \in S} f_{s}$ may be written $\left(f_{s}\right)_{s \in S}$ or just $\left(f_{s}\right)$. We let $\underline{n}$ denote the set $\{1, \ldots, n\}$, our most common indexing set.

Definition 1.1. If $M$ is a smooth manifold and let $C_{n}(M)$ be the subspace of $\left(x_{i}\right) \in M^{\underline{n}}$ such that $x_{i} \neq x_{j}$ if $i \neq j$. Let $\iota$ denote the inclusion of $C_{n}(M)$ in $M \underline{n}$.

Suppose that $M$ were equipped with a metric. The main compactification which we study, $C_{n}[M]$, is homeomorphic to the subspace of $C_{n}(M)$ for which $d\left(x_{i}, x_{j}\right) \geq \epsilon$ for some sufficiently small $\epsilon$. From this model, however, it is not clear how $C_{n}(M)$ should be a subspace of the compactification, much less how to establish functorality or more delicate properties we will develop.

Definition 1.2. For $(i, j) \in C_{2}(\underline{n})$, let $\pi_{i j}: C_{n}\left(\mathbb{R}^{k}\right) \rightarrow S^{k-1}$ be the map which sends $\left(x_{i}\right)$ to the unit vector in the direction of $x_{i}-x_{j}$. Let $I$ be the closed interval from 0 to $\infty$, and for $(i, j, k) \in C_{3}(\underline{n})$ let $s_{i j k}: C_{n}\left(\mathbb{R}^{k}\right) \rightarrow I=[0, \infty]$ be the map which sends $\left(x_{i}\right)$ to $\left(\left|x_{i}-x_{j}\right| /\left|x_{i}-x_{k}\right|\right)$.

Our compactifications are defined as closures, for which we also set notation.

Notation. If $A$ is a subspace of $X$ we let $c l_{X}(A)$, or simply $c l(A)$ if by context $X$ is understood, denote the closure of $A$ in $X$.

From now on by a manifold $M$ we mean a submanifold of some $\mathbb{R}^{k}$, so that $C_{n}(M)$ is a submanifold of $C_{n}\left(\mathbb{R}^{k}\right)$. For $M=\mathbb{R}^{k}$, we specify that $\mathbb{R}^{k}$ is a submanifold of itself through the identity map.

Definition 1.3. Let $A_{n}[M]$, the main ambient space in which we work, be the product $M^{\underline{n}} \times\left(S^{k-1}\right)^{C_{2}(\underline{n})} \times$ $I^{C_{3}(\underline{n})}$, and similarly let $A_{n}\langle[M]\rangle=M^{\underline{n}} \times\left(S^{k-1}\right)^{C_{2}(\underline{n})}$. Let

$$
\alpha_{n}=\iota \times\left(\left.\pi_{i j}\right|_{C_{n}(M)}\right) \times\left(\left.\left(s_{i j k}\right)\right|_{C_{n}(M)}\right): C_{n}(M) \rightarrow A_{n}[M]
$$

and define $C_{n}[M]$ to be $c l_{A_{n}[M]}\left(i m\left(\alpha_{n}\right)\right)$. Similarly, let $\beta_{n}=\iota \times\left(\left.\pi_{i j}\right|_{C_{n}(M)}\right): C_{n}(M) \rightarrow A_{n}\langle[M]\rangle$ and define $C_{n}\langle[M]\rangle$ to be $c l_{A_{n}\langle[M]\rangle}\left(\operatorname{im}\left(\beta_{n}\right)\right)$.

We will show that $C_{n}[M]$ is a manifold with corners whose diffeomorphism type depends only on that of $M$ below. Because $A_{n}[M]$ is compact when $M$ is, and $C_{n}[M]$ is closed in $A_{n}[M]$, we immediately have the following.

Proposition 1.4. If $M$ is compact, $C_{n}[M]$ is compact.

We call $C_{n}[M]$ the canonical compactification of $C_{n}(M)$ and $C_{n}\langle[M]\rangle$ the simplicial variant. When $M$ is not compact but is equipped with a complete metric, it is natural to call $C_{n}[M]$ the canonical completion of $C_{n}(M)$.

1.2. Review of previous work. The compactification $C_{n}[M]$ first appeared in work of Axelrod and Singer [1, who translated the definition of Fulton and MacPherson in [10] as a closure in a product of blow-ups from algebraic geometry to the setting of manifolds using spherical blow-ups. Kontsevich made similar constructions at about the same time as Fulton and MacPherson, and his later definition in [15. coincides with our $\widetilde{C}_{n}\left\langle\left[\mathbb{R}^{k}\right]\right\rangle$, though it seems that he was trying to define $\widetilde{C}_{n}\left[\mathbb{R}^{k}\right]$. Kontsevich's oversight was corrected in [11, in which Gaiffi gives a definition of $C_{n}\left[\mathbb{R}^{k}\right]$ similar to ours, generalizes the construction for arbitrary hyperplane arrangments over the real numbers, gives a pleasant description of the category of strata using the language of blow-ups of posets from [9], and also treats blow-ups for stratified spaces locally and so gives rise to a new definition of $C_{n}[M]$. 
Axelrod and Singer used these compactifications to define invariants of three-manifolds coming from Chern-Simons theory, and these constructions have generally been vital in quantum topology [3, 17, 2, 19]. Extensive use of similar constructions has been made in the setting of hyperplane arrangments [6, 25] over the complex numbers. These compactifications have also inspired new computational results [16, 24, and they canonically realize the homology of $C_{n}\left(\mathbb{R}^{k}\right)[21$. We came to the present definitions of these compactifications so we could define maps and boundary conditions needed for applications to knot theory 4, 22 .

New results include full proofs of many folk theorems, and the following:

- A construction for general manifolds which bypasses the need for blow-ups, uses simple global coordinates, and through which functorality is immediate.

- Explicit description of the strata in terms of spaces of configurations in the tangent bundle.

- Full treatment of the simplicial variant, including a proof that the projection from the canonical compactification to the simplicial one is a homotopy equivalence.

- A clarification of the central role which Stasheff's associahedron plays in this setting.

- Constructions of diagonal maps, projections, and substitution maps as needed for applications. The constructions of these maps are signifcantly aided by having simple global coordinates.

In future work [18, we will use these constructions to define an operad structure on these compactifications of configurations in Euclidean space, which has consequences in knot theory. This operad structure was first applied in [12.

We also hope that a unified and explicit exposition of these compactifications using our simplified definition could be of help, especially to those who are new to the subject.

1.3. A comment on notation, and a little lemma. There are two lines of notation for configuration spaces of manifolds in the literature, namely $C_{n}(M)$ and $F(M, n)$. Persuaded by Bott, we choose to use the $C_{n}(M)$ notation. Note, however, that $C_{n}(M)$ in this paper is $C_{n}^{0}(M)$ in [3] and that $C_{n}[M]$ in this paper is $C_{n}(M)$ in [3]. Indeed, we warn the reader to pay close attention to the parentheses in our notation: $C_{n}(M)$ is the open configuration space; $C_{n}[M]$ is the Fulton-MacPherson/Axelrod-Singer compactification, its canonical completion; $C_{n}\langle[M]\rangle$, the simplicial variant, is a quotient of $C_{n}[M] ; C_{n}\{M\}$, an auxilliary construction, is a subspace of $C_{n}[M]$ containing only one additional stratum. We suggest that those who choose to use $F(M, n)$ for the open configuration space use $F[M, n]$ for the compactification.

As closures are a central part of our definitions, we need a lemma from point-set topology that open maps commute with taking closures.

Lemma 1.5. Let $A$ be a subspace of $X$, and let $\pi: X \rightarrow Y$ be an open map. Then $\pi\left(c l_{X}(A)\right) \subseteq c l_{Y}(\pi(A))$. If $c l_{X}(A)$ is compact (for example, when $X$ is) then this inclusion is an equality.

Proof. First, $\pi^{-1}\left(c l_{Y}(\pi(A))\right)$ is closed in $X$ and contains $A$, so it contains $c l_{X}(A)$ as well. Applying $\pi$ to this containment we see that $\pi\left(c l_{X}(A)\right) \subseteq c l_{Y}(\pi(A))$.

If $c l_{X}(A)$ is compact, so is $\pi\left(c l_{X}(A)\right)$, which is thus closed in $Y$. It contains $\pi(A)$, therefore $c l_{Y}(\pi(A)) \subseteq$ $\pi\left(c l_{X}(A)\right)$.

1.4. Acknowledgements. The author would like to thank Dan Dugger for providing a proof and references for Lemma [5.6] Ismar Volic for working with the author on an early draft of this paper, Matt Miller for a careful reading, and Giovanni Gaiffi and Eva-Maria Feitchner for sharing preprints of their work.

\section{A CATEGORY OF TREES AND RELATED CATEGORIES}

In order to understand the compactifications $C_{n}[M]$ we have to understand their strata, which are naturally labelled by a poset (or category) of trees. 
Definition 2.1. Define an $f$-tree to be a rooted, connected tree, with labelled leaves, and with no bivalent internal vertices. Thus, an $f$-tree $T$ is a connected acyclic graph with a specified vertex $v_{0}$ called the root. The root may have any valence, but other vertices may not be bivalent. The univalent vertices other than perhaps the root are called leaves, and each leaf is labelled uniquely with an element of $\underline{\# l(T)}$, where $l(T)$ is the set of leaves of $T$ and $\# l(T)$ is its cardinality.

Figure 2.2. A tree $T$.

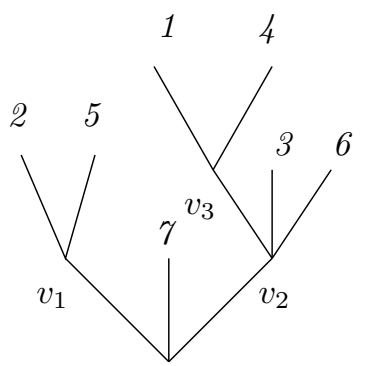

$v_{0}$

In an $f$-tree there is a unique path from any vertex or edge to the root vertex, which we call its root path. We say that one vertex or edge lies over another if the latter is in the root path of the former. For any edge, its boundary vertex closer to the root is called its initial vertex, and its other vertex is called its terminal vertex. If two edges share the same initial vertex we call them coincident. For a vertex $v$ there is a canonical ordering of edges for which $v$ is initial, the collection of which we call $E(v)$, the group of edges coincident at $v$. Namely, set $e<f$ if the smallest label for a leaf over $e$ is smaller than that over $f$. We may use this ordering to name these edges $e_{1}(v), \ldots, e_{\# v}(v)$, where $\# v$ is the number of edges $\mathrm{f}$ in $E(v)$.

We will be interested in the set of $f$-trees as a set of objects in a category in which morphisms are defined by contracting edges.

Definition 2.3. Given an $f$-tree $T$ and a set of non-root, non-leaf edges $E$ the contraction of $T$ by $E$ is the tree $T^{\prime}$ obtained by, for each edge $e \in E$, identifying its initial vertex with its terminal vertex and removing $e$ from the set of edges.

Definition 2.4. Define $\Psi_{\underline{n}}$ to be the category whose objects are $f$-trees with $n$ leaves. There is a (unique) morphism in $\Psi_{\underline{n}}$ from $T$ to $T^{\prime}$ if $T^{\prime}$ is isomorphic to a contraction of $T$ along some set of edges.

Figure 2.5. The category $\Psi_{3}$.

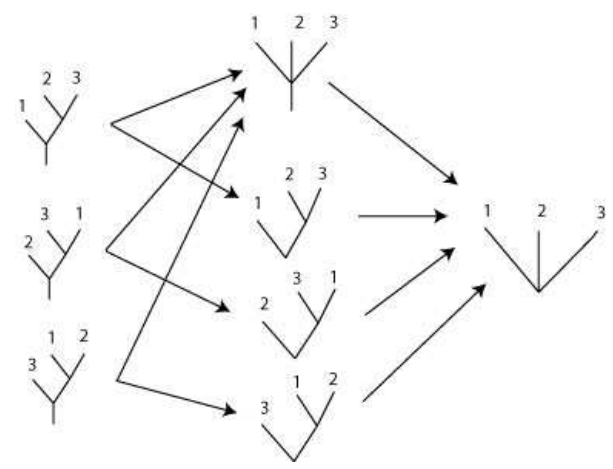

Finally, let $V(T)$ denote the set of non-leaf vertices of $T$. Let $V^{i}(T)$ denote its subset of internal vertices (thus only excluding the root). Note that a morphism in $\Psi_{\underline{n}}$ decreases the number of internal vertices, 
which is zero for the terminal object in $\Psi_{\underline{n}}$. Let $\widetilde{\Psi_{n}}$ be the full subcategory of $f$-trees whose root is univalent (informally, trees with a trunk). Note that $\widetilde{\Psi_{n}}$ has an operad structure, as defined in [12].

It is useful to have facility with categories which are essentially equivalent to $\Psi_{\underline{n}}$. We will define these categories through the notions of parenthesization and exclusion relation. Further equivalent constructions include the collections of screens of Fulton and MacPherson [10]. The best perspective on these categories is given by the combinatorial blow-up of Feitchner and Kozlov [9. Indeed, Gaiffi shows in [11] that the poset of strata of a blow-up of an arrangment is the combinatorial blow-up of the orignial poset associated to the arrangment. Since we focus not on general blow-ups but on compactified configuration spaces in particular, we choose more concrete manifestations of this category.

Definition 2.6. A (partial) parenthesization $\mathcal{P}$ of a set $S$ is a collection $\left\{A_{\alpha}\right\}$ of nested subsets of $S$, each of cardinality greater than one. By nested we mean that for any $\alpha, \beta$ the intersection $A_{\alpha} \cap A_{\beta}$ is either $A_{\alpha}$, $A_{\beta}$ or empty. The parenthesizations of $S$ form a poset, which we call $\operatorname{Pa}(S)$, in which $\mathcal{P} \geq \mathcal{P}^{\prime}$ if $\mathcal{P} \subseteq \mathcal{P}^{\prime}$.

Parenthesizations are related to trees in that they may keep track of sets of leaves which lie over the vertices of a tree.

Definition 2.7. Define $f_{1}: \Psi_{\underline{n}} \rightarrow P a(\underline{n})$ by sending a tree $T$ to the collection of sets $\left\{A_{v}\right\}$, where $v \in V^{i}(T)$ and $A_{v}$ is the set of indices of leaves which lie over $v$. Define $g_{1}: P a(\underline{n}) \rightarrow \Psi_{\underline{n}}$ by sending a parenthesization to a tree with the following data

- One internal vertex $v_{\alpha}$ for each $A_{\alpha}$.

- An edge between $v_{\alpha}$ and $v_{\beta}$ if $A_{\alpha} \subset A_{\beta}$ but there is no proper $A_{\alpha} \subset A_{\gamma} \subset A_{\beta}$.

- A root vertex with an edges connecting it to each internal vertex corresponding to a maximal $A_{\alpha}$.

- Leaves with labels in $\underline{n}$ with an edge connecting the $i$ th leaf to either the vertex $v_{\alpha}$ where $A_{\alpha}$ is is the minimal set containing $i$, or the root vertex of there is no such $A_{\alpha}$.

We leave to the reader the straightforward verification that $f_{1}$ and $g_{1}$ are well-defined and that the following proposition holds.

Proposition 2.8. The functors $f_{1}$ and $g_{1}$ are isomorphisms between the categories $\Psi_{\underline{n}}$ and $P a(\underline{n})$.

Another way in which to account for the data of which leaves lie above common vertices in a tree is through the notion of an exclusion relation.

Definition 2.9. Define an exclusion relation $R$ on a set $S$ to be a subset of $C_{3}(S)$ such that the following properties hold

(1) If $(x, y), z \in R$ then $(y, x), z \in R$ and $(x, z), y \notin R$.

(2) If $(x, y), z \in R$ and $(w, x), y \in R$ then $(w, x), z \in R$.

Let $\operatorname{Ex}(S)$ denote the poset of exclusion relations on $S$, where the ordering is defined by inclusion as subsets of $C_{3}(S)$.

We now construct exclusion relations from parenthesizations, and vice versa.

Definition 2.10. Let $f_{2}: P a(\underline{n}) \rightarrow E x(\underline{n})$ be defined by setting $(i, j), k \in R$ if $i, j \in A_{\alpha}$ but $k \notin A_{\alpha}$ for some $A_{\alpha}$ in the given parenthesization. Define $g_{2}: \operatorname{Ex}(\underline{n}) \rightarrow P a(\underline{n})$ by, given an exclusion relation $R$, taking the collection of sets $A_{\sim i, \neg k}$ where $A_{\sim i, \neg k}$ is the set of all $j$ such that $(i, j), k \in R$, along with $i$ when there is such a $j$. Let $\operatorname{Tr}=g_{1} \circ g_{2}: \operatorname{Ex}(\underline{n}) \rightarrow \Psi_{\underline{n}}$ and let $\mathcal{E} x=f_{2} \circ f_{1}$.

As above, we leave the proof of the following elementary proposition to the reader.

Proposition 2.11. The composite $f_{2} \circ g_{2}$ is the identity functor. If $f_{2}(\mathcal{P})=f_{2}\left(\mathcal{P}^{\prime}\right)$ then $\mathcal{P}$ and $\mathcal{P}^{\prime}$ may only differ by whether or not they contain the set $\underline{n}$ itself. 


\section{The STRATIFICATION OF THE BASIC COMPACTIFICATION}

This section is the keystone of the paper. We first define a stratification of $C_{n}[M]$ through coordinates as a subspace of $A_{n}[M]$. For our purposes, a stratification is any expression of a space as a finite disjoint union of locally closed subspaces called strata, which are usually manifolds, such that the closure of each stratum is its union with other strata. We will show that when $M$ has no boundary, the stratification we define through coordinates coincides with the stratification of $C_{n}[M]$ as a manifold with corners. The strata of $C_{n}[M]$ are individually simple to describe, so constructions and maps on $C_{n}[M]$ are often best understood in terms of these strata.

Before treating $C_{n}[M]$ in general, we would like to be completely explicit about the simplest possible case, essentially $C_{2}\left[\mathbb{R}^{k}\right]$.

Example. Let $C_{2}^{*}\left(\mathbb{R}^{k}\right) \cong \mathbb{R}^{k}-0$ be the subspace of points $(0, x \neq 0) \in C_{2}\left(\mathbb{R}^{k}\right)$ and consider its closure as the subspace of $\mathbb{R}^{k} \times S^{k-1}$ of points $\left(x \neq 0, \frac{x}{\|x\|}\right)$. The projection of this subspace onto $S^{k-1}$ coincides with the tautological positive ray bundle over $S^{k-1}$, which is a trivial bundle. The closure $C_{2}^{*}\left[\mathbb{R}^{k}\right]$ is the non-negative ray bundle, which is diffeomorphic to $S^{k-1} \times[0, \infty)$. Projecting this closure onto $\mathbb{R}^{k}$ is a homeomorphism when restricted to $\mathbb{R}^{k}-0$, and the preimage of 0 is a copy of $S^{k-1}$, the stratum of added points. Thus, $C_{2}^{*}\left[\mathbb{R}^{k}\right]$ is diffeomorphic to the blow-up of $\mathbb{R}^{k}$ at 0 , in which one replaces 0 by the sphere of directions from which it can be approached. Unlike the locally-defined blow-up, $C_{2}^{*}\left[\mathbb{R}^{k}\right]$ has simple global coordinates inherited from $\mathbb{R}^{k} \times S^{k-1}$.

3.1. Stratification of $C_{n}[M]$ using coordinates in $A_{n}[M]$. We proceed to define a stratification for $C_{n}[M]$ by associating an $f$-tree to each point in $C_{n}[M]$.

Definition 3.1. Let $x=\left(\left(x_{i}\right),\left(u_{i j}\right),\left(d_{i j k}\right)\right) \in C_{n}[M]$. Let $R(x)$ be the exclusion relation defined by $(i, j), k \in R(x)$ if $d_{i j k}=0$. Let $T(x)$ be equal to either $\operatorname{Tr}(R(x))$ or, if all of the $x_{i}$ are equal, the $f$-tree obtained by adding a new root to $\operatorname{Tr}(R(x))$.

Note that because $d_{i j k} d_{i \ell j}=d_{i \ell k}$ for points in the image of $C_{n}(M)$, by continuity this is true for all of $C_{n}[M]$. So if $d_{i j k}=0=d_{i \ell j}$ then $d_{i \ell k}=0$. Therefore, $R(x)$ satisfies the last axiom for an exclusion relation. The other axiom is similarly straightforward to check to see that $R(x)$ is well-defined.

Definition 3.2. Let $C_{T}(M)$ denote the subspace of all $x \in C_{n}[M]$ such that $T(x)=T$, and let $C_{T}[M]$ be its closure in $C_{n}[M]$.

The following proposition, which gives a first indication of how the $C_{T}(M)$ fit together, is an immediate consequence of the definitions above.

Proposition 3.3. Let $s=\left\{\left(x_{i}\right)_{j}\right\}_{j=1}^{\infty}$ be a sequence of of points in $C_{n}(M)$ which converges to a point in $C_{n}[M] \subset A_{n}[M]$. The limit of $s$ is in $C_{T}[M]$ if and only if the limit of $d\left(x_{i}, x_{j}\right) / d\left(x_{i}, x_{k}\right)$ approaches zero for every $(i, j), k \in \operatorname{Ex}(T)$ and, in the case where the root valence of $T$ is one, we also have that all of the $x_{i}$ approach the same point in $M$.

To a stratification of a space, one may associate a poset in which stratum $\alpha$ is less than stratum $\beta$ if $\alpha$ is contained in the closure of $\beta$.

Theorem 3.4. The poset associated to the stratification of $C_{n}[M]$ by the $C_{T}(M)$ is isomorphic to $\Psi_{\underline{n}}$.

Proof. This theorem follows from the preceding proposition and the fact that if $T \rightarrow T^{\prime}$ is a morphism in $\Psi_{\underline{n}}$ then $R\left(T^{\prime}\right)$ is contained in $R(T)$.

3.2. Statement of the main theorem. Having established an intrinsic definition for the $C_{T}(M)$ and a combinatorial description of how they fit together, we now set ourselves to the more difficult task of identifying these spaces explicitly. We describe the spaces $C_{T}(M)$ in terms of "infinitesimal configurations". 
Definition 3.5. - Let $\operatorname{Sim}_{k}$ be the subgroup of the group of affine transformations in $\mathbb{R}^{k}$ generated by translation and scaling.

- Define $I C_{i}(M)$ to be the space of $i$ distinct points in $T M$ all lying in one fiber, modulo the action of $\operatorname{Sim}_{k}$ in that fiber. Let $p$ be the projection of $I C_{i}(M)$ onto $M$.

For example $I C_{2}(M)$ is diffeomorphic to $S T M$, the unit tangent bundle of $M$.

Let $e \in E_{0}=E\left(v_{0}\right)$ be a root edge of an $f$-tree $T$, and let $V(e) \subseteq V^{i}(T)$ be the set of internal vertices which lie over $e$.

Definition 3.6. $\quad$ (1) Define $I C_{e}(M)$ to be subspace of the product $\left(I C_{\# v}(M)\right)^{V(e)}$ of tuples of infinitesimal configurations all sitting over the same point in $M$.

(2) Let $p_{e}$ be the map from $I C_{e}(M)$ onto $M$ defined projecting onto that point.

(3) Let $D_{T}(M)$ be the subspace of $\left(I C_{e}(M)\right)^{E_{0}}$ of points whose image under $\left(p_{e}\right)$ in $(M)^{E_{0}}$ sits in $C_{\# v_{0}}(M)$.

In other words, a point in $D_{T}(M)$ is a collection of $\# v_{0}$ distinct points $\left(x_{e}\right)_{e \in E_{0}}$ in $M$ with a collection of $\# v(e)$ infinitesimal configurations at each $x_{e}$.

Figure 3.7. A point in $D_{T}(M)$ with $T$ from Figure 2.2.
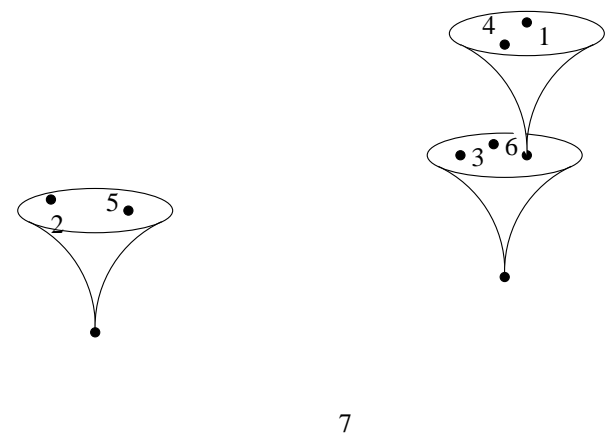

The following theorem is the main theorem of this section.

Theorem 3.8. $C_{T}(M)$ is diffeomorphic to $D_{T}(M)$.

Remark. To intuitively understand $C_{T}(M)$ as part of the boundary of $C_{n}[M]$ one views an element of $I C_{i}(M)$ as a limit of a sequence in $C_{i}(M)$ which approaches a point $(x, x, \ldots x)$ in the (thin) diagonal of $M^{i}$. Eventually, in such a sequence all the points in a configuration would lie in a coordinate neighborhood of $x$, which can through the exponential map can be identified with $T_{x} M$, and the limit is taken in that tangent space up to rescaling. If $i>2, I C_{i}(M)$ is itself not complete, so one allows these infinitesimal configurations to degenerate as well, and this is how the situation is pictured in Figure 3.7 Because $T(T M) \cong \oplus_{3} T M$, the recursive nature of having sub-configurations degenerate is not reflected in the topology of $D_{T}(M)$.

To establish this theorem we focus on the case in which $M$ is Euclidean space $\mathbb{R}^{k}$, as $D_{T}\left(\mathbb{R}^{k}\right)$ admits a simple description.

Definition 3.9. Let $\widetilde{C}_{n}\left(\mathbb{R}^{k}\right)$ be the quotient of $C_{n}\left(\mathbb{R}^{k}\right)$ by $S i m_{k}$ acting diagonally, and let $q$ denote the quotient map. Choose coset representatives to identify $\widetilde{C}_{n}\left(\mathbb{R}^{k}\right)$ with a subspace of $C_{n}\left(\mathbb{R}^{k}\right)$, namely the subspace of points $\left(x_{i}\right)$ whose center of mass is $\overrightarrow{0}$ and such that the maximum of the $d\left(x_{i}, \overrightarrow{0}\right)$ is one.

Because the tangent bundle of $\mathbb{R}^{k}$ is trivial, $I C_{i}\left(\mathbb{R}^{k}\right) \cong \mathbb{R}^{k} \times \widetilde{C}_{i}\left(\mathbb{R}^{k}\right)$, and we have the following. 
Proposition 3.10. $D_{T}\left(\mathbb{R}^{k}\right)=C_{\# v_{0}}\left(\mathbb{R}^{k}\right) \times\left(\widetilde{C}_{\# v}\left(\mathbb{R}^{k}\right)\right)^{V^{i}(T)}$.

Alternately, $D_{T}\left(\mathbb{R}^{k}\right)$ is the space in which each edge in $T$ is assigned a point in $\mathbb{R}^{k}$, with coincident edges assigned distinct points, modulo translation and scaling of coincident groups of edges.

Roughly speaking, the proof of Theorem 3.8 when $M=\mathbb{R}^{k}$ respects the product decomposition of Proposition 3.10 We start by addressing the stratum associated to the tree $\Psi$ with a single internal vertex connected to a univalent root.

\subsection{The auxilliary construction, $C_{n}\left\{\mathbb{R}^{k}\right\}$.}

Definition 3.11. Let $A_{n}\{M\}=(M)^{\underline{n}} \times\left(S^{k-1}\right)^{C_{2}(\underline{n})} \times(0, \infty)^{C_{3}(\underline{n})}$, a subspace of $A_{n}[M]$. Note that the image of $\alpha_{n}: C_{n}(M) \rightarrow A_{n}[M]$ lies in $A_{n}\{M\}$. Let $C_{n}\{M\}$ be $c l_{A_{n}\{M\}}\left(i m\left(\alpha_{n}\right)\right)$.

For our purposes, $C_{n}\{M\}$ will be useful as a subspace of $C_{n}[M]$ to first understand, which we do for $M=\mathbb{R}^{k}$.

Theorem 3.12. $C_{n}\left\{\mathbb{R}^{k}\right\}$ is diffeomorphic to $D_{n}\left\{\mathbb{R}^{k}\right\}=\mathbb{R}^{k} \times \widetilde{C}_{n}\left(\mathbb{R}^{k}\right) \times[0, \infty)$.

As a manifold with boundar $C_{n}\left\{\mathbb{R}^{k}\right\}$ has two strata, namely $\mathbb{R}^{k} \times \widetilde{C}_{n}\left(\mathbb{R}^{k}\right) \times(0, \infty)$, which we will identify with $C_{n}\left(\mathbb{R}^{k}\right)$, and $\mathbb{R}^{k} \times \widetilde{C}_{n}\left(\mathbb{R}^{k}\right) \times 0$, the points added in this closure. We will see that these correspond to $C_{\psi}\left(\mathbb{R}^{k}\right)$ and $C_{\psi}\left(\mathbb{R}^{k}\right)$ respectively.

To prove Theorem 3.12 we define a map $\nu: D_{n}\left\{\mathbb{R}^{k}\right\} \rightarrow A_{n}\left\{\mathbb{R}^{k}\right\}$ and show that it is a homeomorphism onto $C_{n}\left\{\mathbb{R}^{k}\right\}$. The map $\nu$ will essentially be an expansion from the point in $\mathbb{R}^{k}$ of the infinitesimal configuration given by the point in $\widetilde{C}_{n}\left(\mathbb{R}^{k}\right) \subset\left(\mathbb{R}^{k}\right)^{\underline{n}}$.

Definition 3.13. (1) Define $\eta: D_{n}\left\{\mathbb{R}^{k}\right\} \rightarrow\left(\mathbb{R}^{k}\right)^{n}$ by sending $x \times\left(y_{i}\right) \times t$ to $\left(x+t y_{i}\right)$.

(2) Let $p$ denote the projection from $D_{n}\left\{\mathbb{R}^{k}\right\}$ onto $\widetilde{C}_{n}\left(\mathbb{R}^{k}\right)$.

(3) Let $\widetilde{\pi}_{i j}$ and $\widetilde{s}_{i j k}$ denote the maps on $\widetilde{C}_{n}\left(\mathbb{R}^{k}\right)$ which when composed with $q$ give the original $\pi_{i j}$ and $s_{i j k}$.

(4) Finally, define $\nu: D_{n}\left\{\mathbb{R}^{k}\right\} \rightarrow A_{n}\left\{\mathbb{R}^{k}\right\}$ by $\eta \times\left(\widetilde{\pi}_{i j} \circ p\right) \times\left(\widetilde{s}_{i j k} \circ p\right)$.

When $t>0$, the image of $\eta$ is in $C_{n}\left(\mathbb{R}^{k}\right)$, and moreover we have the following.

Proposition 3.14. The map $\left.\nu\right|_{t>0}$ coincides with $\alpha_{n} \circ \eta$, a diffeomorphism from $\mathbb{R}^{k} \times \widetilde{C}_{n}\left(\mathbb{R}^{k}\right) \times(0, \infty)$ onto the image of $\alpha_{n}$.

Proof. For $t>0$, the map $\eta$ satisfies $\widetilde{\pi}_{i j} \circ p=\pi_{i j} \circ \eta$, and similarly $\widetilde{s}_{i j k} \circ p=s_{i j k} \circ \eta$, showing that $\left.\nu\right|_{t>0}$ coincides with $\alpha_{n} \circ \eta$.

The inverse to $\left.\nu\right|_{t>0}$ is the product of: the map which sends $\left(x_{i}\right)$ to its the center of mass, the quotient map $q$ to $\widetilde{C}_{n}\left(\mathbb{R}^{k}\right)$, and the map whose value is the greatest distance from one of the $x_{i}$ to the center of mass. Both $\left.\nu\right|_{t>0}$ and its inverse are clearly smooth.

Corollary 3.15. $\left.\nu\right|_{t=0}$ has image in $C_{n}\left\{\mathbb{R}^{k}\right\}$.

We come to the heart of the matter. Because $C_{n}\left\{\mathbb{R}^{k}\right\}$ is defined as a closure, to identify it more explicitly we must identify a closed subset of $A_{n}\left\{\mathbb{R}^{k}\right\}$, which we do presently. We will apply this case repeatedly in analysis of $C_{n}\left[\mathbb{R}^{k}\right]$.

Definition 3.16. Let $\widetilde{A}_{n}\left[\mathbb{R}^{k}\right]=\left(S^{k-1}\right)^{C_{2}(\underline{n})} \times I^{C_{3}(\underline{n})}$, and let $\widetilde{A}_{n}\left\{\mathbb{R}^{k}\right\}=\left(S^{k-1}\right)^{C_{2}(\underline{n})} \times(0, \infty)^{C_{3}(\underline{n})}$.

Convention. We extend multiplication on $(0, \infty)$ to its closure by setting $a \cdot \infty=\infty$ if $a \neq 0$ and $0 \cdot \infty=1$.

Lemma 3.17. The map $\iota_{n}=\left(\widetilde{\pi}_{i j}\right) \times\left(\widetilde{s}_{i j k}\right): \widetilde{C}_{n}\left(\mathbb{R}^{k}\right) \rightarrow \widetilde{A}_{n}\left[\mathbb{R}^{k}\right]$ is a diffeomorphism onto its image, which is closed as a subspace of $\widetilde{A}_{n}\left\{\mathbb{R}^{k}\right\}$. 
Proof. Collinear configurations up to translation and scaling are cleary determined by their image under one $\widetilde{\pi}_{i j}$ and the $\widetilde{s}_{i j k}$. For non-collinear configurations, we may reconstruct $\mathbf{x}=\left(x_{i}\right)$ from the $u_{i j}=\widetilde{\pi}_{i j}(\mathbf{x})$ and $d_{i j k}=\widetilde{s}_{i j k}(\mathbf{x})$ up to translation and scaling by for example setting $x_{1}=\overrightarrow{0}, x_{2}=u_{12}$ and then $x_{i}=d_{1 i 2} u_{1 i}$ for any $i$. These assigments of $x_{i}$ are smooth functions, so in fact $\iota_{n}$ is a diffeomorphism onto its image.

For the sake of showing that the image of $\iota_{n}$ is closed, as well as use in section 5 we note that $d_{1 i 2}$ can be determined from the $u_{i j}$ by the law of sines. If $\pm u_{i j}, \pm u_{j k}$ and $\pm u_{i k}$ are distinct then,

$$
\frac{\left|x_{i}-x_{j}\right|}{\sqrt{1-\left(u_{k i} \cdot u_{k j}\right)^{2}}}=\frac{\left|x_{j}-x_{k}\right|}{\sqrt{1-\left(u_{i j} \cdot u_{i k}\right)^{2}}}=\frac{\left|x_{i}-x_{k}\right|}{\sqrt{1-\left(u_{j i} \cdot u_{j k}\right)^{2}}} \text {. }
$$

Thus, in most cases $d_{1 i 2}=\sqrt{\frac{1-\left(u_{2 i} \cdot u_{21}\right)^{2}}{1-\left(u_{i 1} \cdot u_{i 2}\right)^{2}}}$. In general, as long as not all points are collinear, the law of sines above can be used repeatedly to determine all $d_{i j k}$ from the $u_{i j}$, which shows that when restricted to non-collinear configurations, $\left(\widetilde{\pi}_{i j}\right)$ itself is injective.

We identify the image of $\iota_{n}$ as the set of all points $\left(u_{i j}\right) \times\left(d_{i j k}\right)$ which satisfy the following conditions needed to consistently define an inverse to $\iota_{n}$ :

(1) $u_{i j}=-u_{j i}$.

(2) $u_{i j}, u_{j k}$ and $u_{i k}$ all lie in the same great circle on $S^{k-1}$, with $u_{i k}$ strictly between $u_{i j}$ and $u_{j k}$.

(3) If $\pm u_{i j}, \pm u_{j k}$ and $\pm u_{i k}$ are distinct then $d_{i j k}=\sqrt{\frac{1-\left(u_{i k} \cdot u_{j k}\right)^{2}}{1-\left(u_{i j} \cdot u_{j k}\right)^{2}}}$

(4) $d_{i j k}$ are non-zero and finite and

$$
d_{i j k} d_{i k j}=1=d_{i j k} d_{j k i} d_{k i j}=d_{i j k} d_{i \ell j} d_{i k \ell} .
$$

We say a condition is closed if the subspace of points which satisfy it is closed. Note that condition 4 follows from condition 3 when the latter applies.

Condition [1 is clearly closed, and condition 4 is a closed condition in $\widetilde{A}_{n}\left\{\mathbb{R}^{k}\right\}$, since we are already assuming that $d_{i j k} \in(0, \infty)$. Condition $\left[\right.$ says that on an open subspace of this image, the $d_{i j k}$ are a function of the $u_{i j}$ and gives no restrictions away from this subspace, and so is also a closed condition. Considering condition [2] it is a closed condition for $u_{i j}, u_{j k}$ and $u_{i k}$ to all lie on a great circle. It is not usually a closed condition for $u_{i k}$ to be strictly between $u_{i j}$ and $u_{j k}$. But by condition 3 if $u_{i j} \neq \pm u_{j k}$ but $u_{i k}=u_{j k}$ then $d_{i j k}=0$, so in fact condition 2 is closed within the points in $\widetilde{A}_{n}\left\{\mathbb{R}^{k}\right\}$ satisfying condition [3

Because $\left.\nu\right|_{t=0}$ is the product of the diagonal map $\mathbb{R}^{k} \rightarrow\left(\mathbb{R}^{k}\right)^{\underline{n}}$, which is a diffeomorphism onto its image, with $\iota_{n}$ we may deduce the following.

Corollary 3.18. $\left.\nu\right|_{t=0}$ is a diffeomorphism onto its image.

We may now finish analysis of $C_{n}\left\{\mathbb{R}^{k}\right\}$.

Proof of Theorem 3.12. Proposition 3.14 and Corollary 3.18 combine to give that $\nu_{T}: D_{T}\left\{\mathbb{R}^{k}\right\} \rightarrow C_{T}\left\{\mathbb{R}^{k}\right\}$ is injective. We thus want to show that it is surjective and has a continuous inverse.

Consider the projection $p$ from $C_{n}\left\{\mathbb{R}^{k}\right\} \subset A_{n}\left\{\mathbb{R}^{k}\right\}$ to $\left(x_{i}\right) \in\left(\mathbb{R}^{k}\right)^{\underline{n}}$. Over $C_{n}\left(\mathbb{R}^{k}\right)$ the image of $\alpha_{n}$ is its graph, which is locally closed, so $p^{-1}\left(C_{n}\left(\mathbb{R}^{k}\right)\right) \cong C_{n}\left(\mathbb{R}^{k}\right)$. If $x_{i}=x_{j}$ but $x_{i} \neq x_{k}$ for some $i, j, k$, continuity of $s_{i j k}$ would force $d_{i j k}=0$, which is not possible in $A_{n}\left\{\mathbb{R}^{k}\right\}$. Thus no points in $C_{n}\left\{\mathbb{R}^{k}\right\}$ lie over such $\left(x_{i}\right)$. Over the diagonal of $\left(\mathbb{R}^{k}\right)^{\underline{n}}$ we know that $C_{n}\left\{\mathbb{R}^{k}\right\}$ contains at least the image of $\left.\nu\right|_{t=0}$. But by Lemma 3.17 we may deduce that this image is closed in $A_{n}\left\{\mathbb{R}^{k}\right\}$ and thus accounts for all of $C_{n}\left\{\mathbb{R}^{k}\right\}$ over the diagonal.

We define an inverse to $\nu_{T}$ according to this decomposition over $\left(\mathbb{R}^{k}\right)^{n}$. For a point in $C_{n}\left(\mathbb{R}^{k}\right)$, the inverse was given in Proposition 3.14 For points over the diagonal $\left(x_{i}=x\right)$ in $\left(\mathbb{R}^{k}\right)^{\underline{n}}$, the inverse is a 
product of: the map which sends such a point to $x \in \mathbb{R}^{k}, \iota_{n}^{-1}$, and the constant map whose image is $0 \in[0, \infty)$. Smoothness of this inverse is straightforward and left to the reader.

3.4. Proof of Theorem 3.8 for $M=\mathbb{R}^{k}$. Analysis of $C_{T}\left(\mathbb{R}^{k}\right)$ parallels that of $C_{n}\left\{\mathbb{R}^{k}\right\}$. A key construction is that of a map $\nu_{T}: N_{T} \rightarrow A_{n}\left[\mathbb{R}^{k}\right]$, where $N_{T} \subset D_{T}\left(\mathbb{R}^{k}\right) \times[0,1)^{V^{i}(T)}$ is a chosen neighborhood of $D_{T}\left(\mathbb{R}^{k}\right) \times\left(t_{v}=0\right)$. Though as mentioned before, $D_{T}\left(\mathbb{R}^{k}\right)$ is a subspace of $\left(\mathbb{R}^{k}\right)^{E(T)}$, we emphasize the role of the vertices of $T$ in the definition of $D_{T}\left(\mathbb{R}^{k}\right)$ by naming coordinates on $\mathbf{x} \in D_{T}\left(\mathbb{R}^{k}\right)$ as $\mathbf{x}=\left(x_{e}^{v}\right)$, where $v \in V(T)$ and $e \in e(V)$. Recall that for each $v \neq v_{0}$ we consider $\widetilde{C}_{\# v}\left(\mathbb{R}^{k}\right)$ as a subspace of $C_{\# v}\left(\mathbb{R}^{k}\right)$ in order to fix each $x_{e}^{v}$ as an element of $\mathbb{R}^{k}$.

Definition 3.19. (1) Let $N_{T}\left(\mathbb{R}^{k}\right)$ be the subset of $D_{T}\left(\mathbb{R}^{k}\right) \times[0,1)^{V^{i}(T)}$ of points $\mathbf{x} \times\left(t_{v}\right)$, where $\mathbf{x}$ can be any point in $D_{T}\left(\mathbb{R}^{k}\right)$, all $t_{v}<r(\mathbf{x})$, defined by

$$
\frac{r(\mathbf{x})}{(1-r(\mathbf{x}))}=\frac{1}{3} \min \left\{d\left(x_{e}^{v}, x_{e^{\prime}}^{v}\right)\right\}, \text { where } v \in V(T), \quad e, e^{\prime} \in E(v) \text {. }
$$

(2) By convention, set $t_{v_{0}}=1$. Let $s_{w}: N_{T} \rightarrow[0,1)$ send $\mathbf{x} \times\left(t_{v}\right)$ to the product of $t_{v}$ for $v$ in the root path of $w$.

(3) For any vertex $v$ of an $f$-tree $T$ define $y_{v}: N_{T}\left(\mathbb{R}^{k}\right) \rightarrow \mathbb{R}^{k}$ inductively by setting $y_{v_{0}}=0$ and $y_{v}(\mathbf{x})=s_{w} x_{e}^{w}+y_{w}(\mathbf{x})$, where $e$ is the edge for which $v$ is terminal and $w$ is the initial vertex of $e$. Define $\eta_{T}: N_{T} \rightarrow\left(\mathbb{R}^{k}\right)^{l(T)}$ to be $\left(y_{\ell}\right)^{l(T)}$.

Figure 3.20. $\eta_{T}$ of the point from Figure 3.7 (and some $t_{v}>0$ ) with all $y_{v}$ indicated.

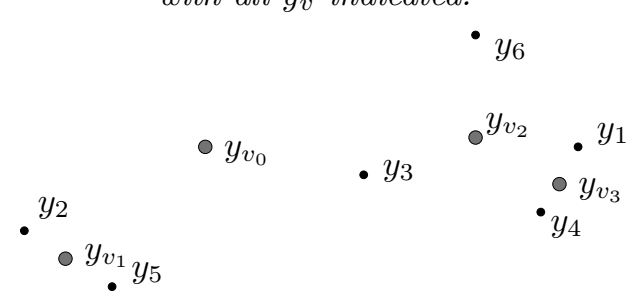

. $y_{7}$

See Figure 3.20 for an illustration of this construction. The most basic case is when $T=V$ the terminal object of $\Psi_{\underline{n}}$, in which case $N_{\Psi}\left(\mathbb{R}^{k}\right)=D_{\Psi}\left(\mathbb{R}^{k}\right)=C_{n}\left(\mathbb{R}^{k}\right)$ and $\eta_{\Downarrow}$ is the canonical inclusion in $\left(\mathbb{R}^{k}\right) \underline{n}$.

Definition 3.21. (1) Given a vertex $w$ of $T$, let $T_{w}$ be the $f$-tree consisting of all vertices and edges over $w$, where $w$ serves as the root of $T_{w}$ and the leaves over $w$ are re-labelled consistent with the order of their labels in $T$.

(2) Let $\rho_{w}: N_{T}\left(\mathbb{R}^{k}\right) \rightarrow N_{T_{w}}\left(\mathbb{R}^{k}\right)$ be the projection onto factors indexed by vertices in $T_{w}$, but with $t_{w}$ set to one and the projection onto $\widetilde{C}_{\# w}\left(\mathbb{R}^{k}\right)$ composed with the canonical inclusion to $C_{\# w}\left(\mathbb{R}^{k}\right)$.

(3) Let $f_{i j}$ be the composite $\pi_{i^{\prime} j^{\prime}} \circ \eta_{T_{v}} \circ \rho_{v}$, where $v$ is the join of the leaves labelled $i$ and $j$, and $i^{\prime}$ and $j^{\prime}$ are the labels of the corresponding leaves of $T_{v}$. Similarly, let $g_{i j k}$ be the composite $s_{i^{\prime} j^{\prime} k^{\prime}} \circ \eta_{T_{w}} \circ \rho_{w}$ where $w$ is the join of leaves $i, j$ and $k$.

(4) Define $\nu_{T}: N_{T} \rightarrow A_{n}\left[\mathbb{R}^{k}\right]$ to be the product $\eta_{T} \times\left(f_{i j}\right) \times\left(g_{i j k}\right)$. Let $\nu_{T}^{0}$ be the restriction of $\nu_{T}$ to $D_{T} \times(0)^{n} \subset N$.

Proposition 3.22. The image of $D_{T}\left(\mathbb{R}^{k}\right)$ under $\nu_{T}^{0}$ lies in $C_{T}\left(\mathbb{R}^{k}\right)$.

Proof. First note that $\left.\eta_{T}\right|_{\left(t_{i}>0\right)}$ has image in $C_{n}\left(\mathbb{R}^{k}\right)$. Moreover, if all $t_{i}>0, f_{i j}$ coincides with $\pi_{i j} \circ \eta_{T}$ and similarly $g_{i j k}=s_{i j k} \circ \eta_{T}$. Thus, the image of $\left.\nu_{T}\right|_{\left(t_{i}>0\right)}$ lies in $\alpha_{n}\left(C_{n}(M)\right)$, which implies that all of the image of $\nu_{T}$, and in particular that of $\nu_{T}^{0}$, lies in $C_{n}[M]$. 
If $v$ is the join of leaves $i, j$ and $k$ and we set $\left(y_{i}\right)=\nu_{T_{v}}^{0} \circ \rho_{v}\left(\mathbf{x},\left(t_{v}\right)\right)$ then $y_{i^{\prime}}=y_{j^{\prime}}$ and thus $d_{i j k}=$ $s_{i j k}\left(\left(y_{i}\right)\right)=0$ if and only if the join of leaves $i$ and $j$ is some vertex which lies (strictly) over $v$. Thus, the exclusion relation for $\nu_{T}^{0}\left(\mathbf{x},\left(t_{v}\right)\right)$ as an element of $C_{n}[M]$ is the exclusion relation associated to $T$.

The simplest way to see that $\nu_{T}^{0}$ is a homeomorphism onto $C_{T}\left(\mathbb{R}^{k}\right)$ is to decompose it as a product and use our analysis of $C_{n}\left\{\mathbb{R}^{k}\right\}$ to help define an inverse.

Definition 3.23. Let $A_{T}[M] \subset A_{n}\left[\mathbb{R}^{k}\right]$ be the subset of points $\left(x_{i}\right) \times\left(u_{i j}\right) \times\left(d_{i j k}\right)$ such that

- If the join of leaves $i$ and $j$ is not the root vertex then $x_{i}=x_{j}$.

- If $(i, j), k$ is in the exclusion relation $\mathcal{E} x(T)$ then $d_{i j k}=0, d_{i k j}=\infty, d_{k i j}=1$, etc. and $u_{i k}=u_{j k}$.

Let $A_{T}\{M\}$ be the subspace of $A_{T}[M]$ for which if there are no exclusions among $i, j$ and $k$ then $d_{i j k}$ is non-zero and finite.

We claim that $C_{T}\left(\mathbb{R}^{k}\right)=C_{n}\left[\mathbb{R}^{k}\right] \cap A_{T}\left\{\mathbb{R}^{k}\right\}$. The relations between the $x_{i}, u_{i j}$ and $d_{i j k}$ which hold on $C_{n}\left(\mathbb{R}^{k}\right)$ also hold on $C_{T}\left(\mathbb{R}^{k}\right)$ by continuity. Therefore, the defining conditions of $A_{T}\left\{\mathbb{R}^{k}\right\}$ when restricted to its intersection with $C_{n}\left[\mathbb{R}^{k}\right]$ will follow from the conditions $d_{i j k}=0$ when $(i, j) k \in \mathcal{E} x(T)$, which in turn are the only defining conditions for $C_{T}\left[\mathbb{R}^{k}\right]$.

Thus the image of $\nu_{T}^{0}$ lies in $A_{T}\left\{\mathbb{R}^{k}\right\}$. By accounting for diagonal subspaces and reordering terms, we will decompose $\nu_{T}^{0}$ is a product of maps in order to define its inverse. We first set some notation.

Definition 3.24. Given a map of sets $\sigma: R \rightarrow S$ let $p_{\sigma}^{X}$, or just $p_{\sigma}$, denote the map from $X^{S}$ to $X^{R}$ which sends $\left(x_{i}\right)_{i \in S}$ to $\left(x_{\sigma(j)}\right)_{j \in R}$.

Definition 3.25. (1) Given a tree $T$ choose $\sigma_{0}: \# v_{0} \rightarrow \underline{n}$ to be an inclusion of sets such that each point in the image labels a leaf which lies over a distinct root edge of $T$.

(2) Similarly, choose $\sigma_{v}: \underline{\# v} \rightarrow \underline{n}$ to be an inclusion whose image labels leaves which lie over distinct edges for which $v$ is initial.

(3) Let $p_{v_{0}}: A_{n}\left[\mathbb{R}^{k}\right] \rightarrow A_{\# v_{0}}\left[\mathbb{R}^{k}\right]$ be the projection $p_{\sigma_{0}} \times p_{C_{2}\left(\sigma_{0}\right)} \times p_{C_{3}\left(\sigma_{0}\right)}$.

(4) Similarly, let $p_{v}: A_{n}\left[\mathbb{R}^{k}\right] \rightarrow \widetilde{A}_{\# v}\left[\mathbb{R}^{k}\right]$ be the projection $* \times p_{C_{2}\left(\sigma_{v}\right)} \times p_{C_{3}\left(\sigma_{v}\right)}$.

(5) Let $p_{T}$ be the product $\left(p_{v}\right)^{V(T)}$.

For example, with $T$ as in Figure 2.2 the image of $\sigma_{0}$ could be $\{5,7,4\}$ and of $\sigma_{v_{1}}$ could be $\{2,5\}$.

Proposition 3.26. For any choices of $\sigma_{v}$, the projection $p_{T}$ restricted to $A_{T}\left[\mathbb{R}^{k}\right]$ is a diffeomorphism onto $A_{\# v_{0}}\left[\mathbb{R}^{k}\right] \times\left(\widetilde{A}_{\# v}\left(\mathbb{R}^{k}\right)\right)^{V^{i}(T)}$, splitting the inclusion of $A_{T}\left[\mathbb{R}^{k}\right]$ in $A_{n}\left[\mathbb{R}^{k}\right]$. Moreover, composed with this diffeomorphism, $\nu_{T}^{0}$ is the product $\alpha_{\# v_{0}} \times\left(\iota_{\# v}\right)$. Analogous results hold for $A_{T}\left\{\mathbb{R}^{k}\right\}$.

We leave the proof of this proposition, which is essentially unraveling defintions, to the reader. We will now define the inverse to $\nu_{T}^{0}$ one vertex at a time. For $v \in V^{i}(T)$, consder $p_{v}(y) \in \widetilde{A}_{\# v}\left\{\mathbb{R}^{k}\right\}$, which by Lemma 1.5 lies in the closure of the image of $\left.p_{v}\right|_{C_{n}\left(\mathbb{R}^{k}\right)}$. The image of $\left.p_{v}\right|_{\left.C_{n}\left(\mathbb{R}^{k}\right)\right)}$ coincides with the image of $\iota_{\# v}$, and by Lemma 3.17 the image of $\iota_{\# v}$ is already closed in $\widetilde{A}_{\# v}\left\{\mathbb{R}^{k}\right\}$. Moreover, $\iota_{\# v}$ is a diffeomorphism onto its image, so we may define the following.

Definition 3.27. (1) For $v \in V^{i}(T)$, let $\phi_{v}: C_{T}\left(\mathbb{R}^{k}\right) \rightarrow \widetilde{C}_{\# v}\left(\mathbb{R}^{k}\right)=\iota_{\# v}^{-1} \circ p_{v}$.

(2) For $v_{0}$, note that if $y \in C_{T}\left(\mathbb{R}^{k}\right)$ then $p_{v_{0}}(y)$ lies in the image of $\alpha_{\# v_{0}}$. Define $\phi_{v_{0}}=\alpha_{\# v_{0}}^{-1} \circ p_{v_{0}}$.

(3) Let $\phi_{T}=\left(\phi_{v}\right)^{v \in V(T)}: C_{T}\left(\mathbb{R}^{k}\right) \rightarrow C_{\# v_{0}}\left(\mathbb{R}^{k}\right) \times\left(C_{\# v}\left(\mathbb{R}^{k}\right)\right)^{V^{i}(T)}$. 
In other words, $\phi_{T}$ is the composite

$$
\begin{aligned}
C_{T}\left(\mathbb{R}^{k}\right) \subset A_{T}\left\{\mathbb{R}^{k}\right\} \stackrel{p_{v}}{\longrightarrow} A_{\# v_{0}}\left\{\mathbb{R}^{k}\right\} \times\left(\widetilde{A}_{\# v}\left\{\mathbb{R}^{k}\right\}\right)^{V^{i}(T)} & \stackrel{\alpha_{\# v_{0}}^{-1} \times\left(\stackrel{\left(\iota_{\# v}^{-1}\right) V^{i}(T)}{\longrightarrow}\right.}{\longrightarrow} C_{\# v_{0}}\left(\mathbb{R}^{k}\right) \times\left(\widetilde{C}_{\# v}\left(\mathbb{R}^{k}\right)\right)^{V^{i}(T)}=D_{T}\left(\mathbb{R}^{k}\right) .
\end{aligned}
$$

Proof of Theorem 3.8 for $M=\mathbb{R}^{k}$. By Proposition $3.22 \nu_{T}^{0}$ sends $D_{T}\left(\mathbb{R}^{k}\right)$ to $C_{T}\left(\mathbb{R}^{k}\right) \subset A_{n}\left[\mathbb{R}^{k}\right]$. Definition 3.27 constructs $\phi_{T}: C_{T}\left(\mathbb{R}^{k}\right) \rightarrow D_{T}\left(\mathbb{R}^{k}\right)$. By construction, and appeal to Lemma 3.17] they are inverse to one another. We also need to check that $\nu_{T}^{0}$ and $\left(\phi_{v}\right)$ are smooth, which follows by checking that their component functions only involve addition, projection and $\iota_{n}^{-1}$ which we know is smooth from Lemma 3.17

3.5. Proof of Theorem 3.8 for general $M$. To establish Theorem 3.8 for general $M$ we first identify $D_{T}(M)$ as a subspace of $D_{T}\left(\mathbb{R}^{k}\right)$, and then we will make use of the established diffeomorphism between $D_{T}\left(\mathbb{R}^{k}\right)$ and $C_{T}\left(\mathbb{R}^{k}\right)$. To set notation, let $\epsilon$ be the given embeddding of $M$ in $\mathbb{R}^{k}$.

Proposition 3.28. The subspace $I D_{n}(M)$ of $M \times \widetilde{C}_{n}\left(\mathbb{R}^{k}\right)$ consisting of all $(m, \mathbf{x})$ such that all $\pi_{i j}(\mathbf{x})$ are in $T_{m} M \subset \mathbb{R}^{k}$ is diffeomorphic, as a bundle over $M$, to $I C_{n}(M)$. Through these diffeomorphisms, $D_{T}(M)$ is a subspace of $D_{T}\left(\mathbb{R}^{k}\right)$.

Proof sketch. The first statement follows from the standard identification of $T M$ as a sub-bundle of $\left.T \mathbb{R}^{k}\right|_{M}$.

The second statement follows from the first statement and Definition 3.6 of $D_{T}(M)$.

From now on, we identify $D_{T}(M)$ with this subspace of $D_{T}\left(\mathbb{R}^{k}\right)$.

Proposition 3.29. $C_{T}(M) \subseteq \nu_{T}^{0}\left(D_{T}(M)\right)$.

Proof. Since $C_{T}(M)$ is already a subspace of $C_{T}\left[\mathbb{R}^{k}\right]$, we just need to check that its points satisfy the condition of Proposition 3.28 Looking at $\left(x_{i}\right) \times\left(u_{i j}\right) \times\left(d_{i j k}\right) \in C_{n}(M)$ inside $A_{n}[M]$ we see that the $u_{i j}$ are vectors which are secant to $M$. Thus, in the closure, if $x_{i}=x_{j}$ then $u_{i j}$ is tangent to $M$ at $x_{i}$.

To prove the converse to this proposition, we show that $\nu_{T}^{0}\left(D_{T}(M)\right)$ lies in $C_{T}(M)$ by modifying the maps $\eta_{T}$ and $\nu_{T}$ so that the image of the latter is in the image of $\alpha_{n}$. The easily remedied defect of $\eta_{T}$ is that it maps to the tangent bundle of $M$ in $\mathbb{R}^{k}$, not to $M$ itself.

Definition 3.30. - Let $N_{T}(M) \subset N_{T}\left(\mathbb{R}^{k}\right)$ be the subspace of $\left(\mathbf{x},\left(t_{v}\right)\right)$ with $\mathbf{x} \in D_{T}(M)$.

- Let $\eta_{T}^{*}: N_{T}(M) \rightarrow\left(\mathbb{R}^{k}\right)^{n} \times\left(\mathbb{R}^{k}\right)^{n}=T\left(\mathbb{R}^{k}\right)^{n}$ send $\left(\mathbf{x},\left(t_{v}\right)\right)$ to $\eta_{T}(\mathbf{x},(0)) \times \eta_{T}\left(\mathbf{x},\left(t_{v}\right)\right)$.

Proposition 3.31. The image of $\eta_{T}^{*}$ lies in $T \epsilon^{n}: T M^{n} \subset T\left(\mathbb{R}^{k}\right)^{n}$.

Proof. $\eta_{T}\left(\mathbf{x},\left(t_{v}\right)\right)$ is defined by adding vectors which by Proposition 3.28 are tangent to $M$ to the coordinates of $\eta_{T}\left(\mathbf{x},\left(t_{v}\right)\right)$, which are in $M$.

We map to $M \underline{n}$ by composing with the exponential map $\operatorname{Exp}(M)$. For each $\mathbf{x} \in D_{T}(M)$ let $U_{\mathbf{x}}$ be a neighborhood of $\mathbf{x} \times 0$ in $N_{T}(M)$ such that the exponential map $\operatorname{Exp}\left(M^{\underline{n}}\right)$ is injective on $\eta_{T}^{*}\left(U_{\mathbf{x}}\right)$.

Definition 3.32. - Let $\eta_{T}^{M, \mathbf{x}}: U_{\mathbf{x}} \rightarrow M^{\underline{n}}$ be the composite $\operatorname{Exp}\left(M^{\underline{n}}\right) \circ\left(T \epsilon^{n}\right)^{-1} \circ \eta_{T}^{*}$.

- Define $f_{i j}^{M, \mathbf{x}}$ by letting $\left(z_{i}\right)$ denote $\eta_{T}^{M, \mathbf{x}}\left(\mathbf{y},\left(t_{v}\right)\right)$ and setting $f_{i j}^{M, \mathbf{x}}=\pi_{i j} \circ \eta_{T}^{M, \mathbf{x}}$ if $z_{i} \neq z_{j}$ or $D E x p \circ f_{i j}$, where DExp is the derivative of the exponential map at $z_{i} \in T M$ and the composite is well defined since $T M \subset T \mathbb{R}^{k}$.

- Define $\nu_{T}^{M, \mathbf{x}}: U_{\mathbf{x}} \rightarrow A_{n}[M]$ as the product $\eta_{T}^{M, \mathbf{x}} \times\left(f_{i j}^{M}\right) \times\left(s_{i j k} \circ \eta_{T}^{M, \mathbf{x}}\right)$.

By construction, $\left.\nu_{T}^{M, \mathbf{x}}\right|_{\left(t_{i}>0\right)}$ has image in which lies in the image of $\alpha_{n}$ in $A_{n}[M]$. On $D_{T}(M) \cap U_{\mathbf{x}}$ the map $\nu_{T}^{M, \mathbf{x}}$ coincides with $\nu_{T}^{0}$ establishing that $\nu_{T}^{0}\left(D_{T}(M)\right) \subset C_{T}(M)$. Along with Proposition 3.29 and the fact that $\nu_{T}^{0}$ and its inverse are smooth, this completes the proof of Theorem 3.8 


\section{First PROPERTIES}

Having proven Theorem 3.8 we derive some first consequences from both the theorem itself and the arguments which went into its proof.

4.1. Characterization in $A_{n}[M]$ and standard projections. To map from $C_{n}[M]$ as we have defined it one may simply restrict maps from $A_{n}[M]$. To map into $C_{n}[M]$ is more difficult, but the following theorem gives conditions to verify that some point in $A_{n}[M]$ lies in $C_{n}[M]$.

Theorem 4.1. $C_{n}\left[\mathbb{R}^{k}\right]$ is the subspace of $A_{n}\left[\mathbb{R}^{k}\right]$ of points $\left(x_{i}\right) \times\left(u_{i j}\right) \times\left(d_{i j k}\right)$ such that

(1) If $x_{i} \neq x_{k}$ then $u_{i j}=\frac{x_{i}-x_{k}}{\left\|x_{i}-x_{k}\right\|}$ and $d_{i j k}=\frac{d\left(x_{i}, x_{j}\right)}{d\left(x_{i}, x_{k}\right)}$.

(2) If $\pm u_{i j}, \pm u_{j k}$, and $\pm u_{i k}$ are all distinct then $d_{i j k}=\sqrt{\frac{1-\left(u_{k i} \cdot u_{k j}\right)^{2}}{1-\left(u_{j i} \cdot u_{j k}\right)^{2}}}$. Otherwise, if $u_{i k}=u_{j k} \neq u_{i j}$ then $d_{i j k}=0$.

(3) $u_{i j}=-u_{j i}$, and $u_{i j}, u_{i k}$ and $u_{j k}$ are linearly dependent $S^{k-1}$ with $u_{i j}$ between $u_{k j}$ and $u_{i k}$ on a great circle in $S^{k-1}$.

(4) $d_{i j k} d_{i k j}, d_{i j k} d_{i k l} d_{i l j}$ and $d_{i j k} d_{j k i} d_{k i j}$ are all equal to one.

Moreover, $C_{n}[M]$ is the subspace of $C_{n}\left[\mathbb{R}^{k}\right]$ where all $x_{i} \in M$ and if $x_{i}=x_{j}$ then $u_{i j}$ is tangent to $M$ at $x_{i}$.

Proof. It is simple to check that $C_{n}\left[\mathbb{R}^{k}\right]$ satisfies all of the properties listed. In most cases, the properties are given by equalities which hold on $C_{n}\left(\mathbb{R}^{k}\right)$ and thus $C_{n}\left[\mathbb{R}^{k}\right]$ by continuity. We noted in Proposition 3.29 that if $x_{i}=x_{j}$ in $C_{n}[M]$ then $u_{i j}$ is tangent to $M$.

Conversely, we can start with a point $x$ which satisfies these properties, and condition 4 allows us to define $T(x)$ as in Definition [3.1] We can then either mimic the construction of $\eta_{T(x)}$ to find points in the image of $\alpha_{n}$ nearby showing that $x \in C_{n}[M]$, or go through the arguments of section 3 to find an element of $D_{T}(M)$ which maps to $x$ under $\nu_{0}^{T}$. The latter argument proceeds by showing that $x$ lies in $A_{T(x)}\left[\mathbb{R}^{k}\right]$, as we may use the contrapositives to conditions 1 and 2 along with 3 to show show that if $d_{i j k}=0$ then $x_{i}=x_{j}$ and $u_{i k}=u_{j k}$. Then, $p_{v_{0}}(x)$ lies in the image of $\alpha_{\# v_{0}}$ essentially by condition 1 Next, $p_{v}(x)$ lies in the image of $\iota_{\# v}$ by conditions 2 , 3 and 4 as these conditions coincide with those given for the image of $\iota$ in Lemma 3.17. We apply the product $\left(\phi_{v}\right)$ to get a point in $D_{T}\left(\mathbb{R}^{k}\right)$ which maps to $x$ under $\nu_{T}^{0}$.

We next turn our attention to the standard projection maps.

Theorem 4.2. By restricting the projection of $A_{n}[M]$ onto $(M)^{n}$ to $C_{n}[M]$, we obtain a projection map $p$ which is onto, which extends the inclusion ८ of $C_{n}(M)$ in $(M)^{n}$ and for which every point in $C_{n}(M)$ has only one pre-image.

Proof. The fact that $p$ is onto can be seen through composing $p$ with the maps $\nu_{T}^{0}$. It is immediate from definitions that $p$ extends $\iota$. Finally, by our characterization in Theorem 4.1 in particular condition 1 any point in $C_{n}[M]$ which projects to $C_{n}(M)$ will be in the image of $\alpha_{n}$.

When $M=\mathbb{R}^{k}$ it is meaningful to project onto other factors of $A_{n}\left[\mathbb{R}^{k}\right]$ to get similar extensions.

Theorem 4.3. The maps $\pi_{i j}$ and $s_{i j k}$ extend to maps from $C_{n}\left[\mathbb{R}^{k}\right]$. Moreover, the extension $\pi_{i j}$ is an open map.

Proof. The only statement which is not immediate is that $\pi_{i j}$ is an open map. We check this on each stratum, using the identification of $C_{T}\left(\mathbb{R}^{k}\right)$ as a product to see that when $\pi_{i j}$ is restricted to $C_{T}\left(\mathbb{R}^{k}\right)$ it factors as $p_{v}$, where $v$ is the join of leaves $i$ and $j$, composed with some $\tilde{\pi}_{i^{\prime} j^{\prime}}$, each of which is an open map. 
4.2. Manifold structure, codimensions of strata, functoriality for embeddings, and equivariance.

Theorem 4.4. $C_{n}[M]$ is a manifold with corners for which the $\nu_{T}^{M, \mathrm{x}}$ may serve as charts.

Proof. The domains of $\nu_{T}^{M, \mathbf{x}}$ are manifolds with corners, so it suffices to check that these maps are diffeomorphisms onto their images in $A_{n}[M]$, which is itself a manifold with corners. We have already noted that $\nu_{T}^{M, \mathbf{x}}$ are smooth on their domain, as they are defined using addition in $\mathbb{R}^{k}$, projection maps, and the exponential map. Moreover, they may be extended using the same formulas to values of $t_{v}<0$, as needed for smoothness with corners.

For $M=\mathbb{R}^{k}$, the inverse to $\nu_{T}$ is relatively straightforward to define. Given $\mathbf{x}=\left(x_{i}\right) \times\left(u_{i j}\right) \times\left(d_{i j k}\right) \in$ $A_{n}\left[\mathbb{R}^{k}\right]$, first recursively set $y_{v}$ to be the average of $y_{w}$, where $w$ are terminal vertices for edges coincident at $v$, starting with $y_{l}=x_{i}$ when $l$ is the leaf labelled by $i$. We let $\left(y_{v}\right)$, as $v$ ranges over terminal vertices for root edges of $T$, define $\mathbf{x}_{v_{0}} \in C_{\# v_{0}}\left(\mathbb{R}^{k}\right)$.

Along the same lines, for each vertex $v$ first define a point in $\left(\mathbb{R}^{k}\right)^{l\left(T_{v}\right)} / \operatorname{Sim}_{k}$ by, as in the defintion of $\iota_{n}^{-1}$, setting some $x_{i}=0$, some $x_{k}=u_{i k}$ and the rest of the $x_{j}$ as $d_{i j k} x_{i j}$. Recursively set $x_{w}$ to be the average of $x_{u}$ for $u$ directly over $w$ (which is well-defined up to translation and scaling) and let $\left(x_{w}\right)$ as $w$ ranges over vertices directly over $v$ define $\mathbf{x}_{v} \in \widetilde{C}_{\# v}\left(\mathbb{R}^{k}\right)$.

Finally, to compute $t_{w}$ we look within the construction above of $\mathbf{x}_{v}$ for the vertex $v$ over which $w$ sits directly. Let $d_{w}$ be the greatest distance from one of the of $x_{u}$, for $u$ over $w$, to $x_{w}$, and define $d_{v}$ similarly. Set $t_{w}$ to be $d_{w} / d_{v}$.

The map which sends $\mathbf{x}$ as above to $\left(\mathbf{x}_{v}\right) \times\left(t_{v}\right)$ is the inverse to $\nu_{T}$, and it is smooth, defined by averaging and greatest distance functions. The construction for general $M$ works similarly, by first composing with the inverse to the exponential map. We leave its construction to the reader.

Since a manifold with corners is a topological manifold with boundary, and a topological with boundary are homotopy equivalent to their interiors, we get the following.

Corollary 4.5. The inclusion of $C_{n}(M)$ into $C_{n}[M]$ is a homotopy equivlance.

An essential piece of data for a manifold with corners are the dimensions of the strata. Dimension counting for $D_{T}(M)$ leads to the following.

Proposition 4.6. The codimension of $C_{T}(M)$ is $\# V^{i}(T)$.

Contrast this with the image of the projection of $C_{T}(M)$ in $M^{\underline{n}}$, which has codimension equal to $k \cdot \operatorname{dim}(M)$, where $k$ is the sum over all root edges $e$ of $n_{e}-1$ where $n_{e}$ is the number of leaves over $e$.

Next, we have the following long-promised result.

Theorem 4.7. Up to diffeomorphism, $C_{n}[M]$ is independent of the embedding of $M$ in $\mathbb{R}^{k}$.

Proof. The definitions of $D_{T}(M)$ and $N_{T}(M)$ and do not use the embedding of $M$ in $\mathbb{R}^{k}$. Let $f$ and $g$ be two embeddings of $M$ in $\mathbb{R}^{k}$, and let $\nu_{T}^{f, \underline{\mathbf{x}}}$ and $\nu_{T}^{g, \underline{\mathbf{x}}}$ denote the respective versions of $\nu_{T}^{M, \underline{\mathbf{x}}}$. Then the $\nu_{T}^{f, \underline{\mathbf{x}}} \circ\left(\nu_{T}^{g, \underline{\mathbf{x}}}\right)^{-1}$ compatibly define a diffeomorphism between the two versions of $C_{n}[M]$.

In fact, since the exponential maps from $T(M)^{n}$ to $(M)^{n}$ are independent of embedding, so are $\nu_{T}^{M, \underline{\mathbf{x}}}$. Thus, we could use the $\nu_{T}^{M, \underline{\mathbf{x}}}$ to topologize the union of the $C_{T}(M)$ without reference to $A_{n}[M]$. Yet another approach would be to first develop $C_{n}\left[\mathbb{R}^{k}\right]$ and then use a diffeomorphism result Theorem 4.7 to patch $C_{n}[M]$ together from $C_{m}\left[U_{i}\right]$ for $m \leq n$, where $U_{i}$ is a system of charts for $M$.

Corollary 4.8. $C_{n}[-]$ is functorial in that an embedding $f: M \rightarrow N$ induces an embedding of manifolds with corners $C_{n}[f]: C_{n}[M] \rightarrow C_{n}[N]$ which respects the stratifications. Moreover, $C_{T}(M)$ is mapped to $C_{T}(N)$ by $D f$ on each factor of $I C_{i}(M)$. 
Proof. Since we are free to choose the embedding of $M$ in $\mathbb{R}^{k}$ to define $C_{n}[M]$ we may simply compose the chosen embedding of $N$ in $\mathbb{R}^{k}$ with $f$, giving immediately that $C_{n}[M]$ is a subspace of $C_{n}[N]$. Moreover, by definition of the stratification according to conditions of $d_{i j k}=0, C_{T}(M)$ is a subspace of $C_{T}(M)$. The fact that $C_{n}[M]$ is embedded as a submanifold with corners is readily checked on each stratum, using the fact that $I C_{i}(M)$ is a submanifold of $I C_{i}(N)$ through $D f$.

An alternate notation for $C_{n}[f]$ is $e v_{n}(f)$ as it extends the evaluation maps on $C_{n}(M)$ and $M^{\underline{n}}$.

Corollary 4.9. The group of diffeomorphisms of $M$ acts on $C_{n}[M]$, extending and lifting its actions on $C_{n}(M)$ and $M \underline{n}$.

The construction of $C_{n}[M]$ is also compatible with the free symmertric group action $C_{n}(M)$.

Theorem 4.10. The $\Sigma_{n}$ action on $C_{n}(M)$ extends to one on $C_{n}[M]$, which is free and permutes the strata by diffeomorphisms according to the $\Sigma_{n}$ action on $\Psi_{\underline{n}}$. Thus, the quotient $C_{n}[M] / \Sigma_{n}$ is itself a manifold with corners whose category of strata is isomorphic to $\widetilde{\Psi}_{n}$, the category of unlabelled $f$-trees.

Proof. The $\Sigma_{n}$ action on $C_{n}[M]$ may in fact be defined as the restriction of the action on $A_{n}[M]$ given by permutation of indices.

The fact that this action is free follows either from a stratum-by-stratum analysis or, more directly, from the fact that if $\sigma$ is a permutation with a cycle $\left(i_{1}, \ldots, i_{k}\right)$ with $k>1$ and if $u_{i_{1} i_{2}}=u_{i_{2} i_{3}}=\cdots=u_{i_{k-1} i_{k}}=u$ then $u_{i_{1} i_{k}}=u$ as well by condition 3 of Theorem 4.1. This implies that $u_{i_{k} i_{1}}=-u \neq u$, which means that $\sigma$ cannot fix a point in $C_{n}[M]$ unless it is the identity.

Finally, note that the coordinate charts $\nu_{T}^{M, \underline{\mathbf{x}}}$ commute with permutation of indices, so that $\sigma C_{T}(M)=$ $C_{\sigma T}(M)$ through a diffeomorphism, giving rise to a manifold structure on the quotient.

4.3. The closures of strata. We will now see that the passage from the stratum $C_{T}(M)$ to its closure, which by Theorem 3.4 consist of the union of $C_{S}(M)$ for $S$ with a morphism to $T$, is similar to the construction of $C_{n}[M]$ itself.

Definition 4.11. Let $\widetilde{C}_{n}\left[\mathbb{R}^{k}\right]$ be defined as the closure of $\widetilde{C}_{n}\left(\mathbb{R}^{k}\right)$ in $\widetilde{A}_{n}\left[\mathbb{R}^{k}\right]$.

Because $\widetilde{A}_{n}\left[\mathbb{R}^{k}\right]$ is compact, so is $\widetilde{C}_{n}\left[\mathbb{R}^{k}\right]$. We give an alternate construction of this space as follows. Extend the action of $\operatorname{Sim}_{k}$ on $\left(\mathbb{R}^{k}\right)^{\underline{n}}$ to $A_{n}\left[\mathbb{R}^{k}\right]$ by acting trivially on the factors of $S^{k-1}$ and $I$. This action preserves the image of $\alpha_{n}$ and so passes to an action on $C_{n}\left[\mathbb{R}^{k}\right]$. This is a special case of Corollary 4.9] Let $A_{n}\left[\mathbb{R}^{k}\right] / \sim$ and $C_{n}\left[\mathbb{R}^{k}\right] / \sim$ denote the quotients by these actions.

Lemma 4.12. $\widetilde{C}_{n}\left[\mathbb{R}^{k}\right]$ is diffeomorphic to $C_{n}\left[\mathbb{R}^{k}\right] / \sim$.

Proof. First note that $A_{n}\left[\mathbb{R}^{k}\right] / \sim$ is compact, and thus so is $C_{n}\left[\mathbb{R}^{k}\right] / \sim$. The projection map from $A_{n}\left[\mathbb{R}^{k}\right] / \sim$ to $\widetilde{A}_{n}\left[\mathbb{R}^{k}\right]$ thus sends sends $C_{n}\left[\mathbb{R}^{k}\right] / \sim$ onto $\widetilde{C}_{n}\left[\mathbb{R}^{k}\right]$ by Lemma 1.5 In the other direction, we may essentially use the maps $\iota_{k}^{-1}$ to define an inverse to this projection, by reconstructing a point in $\left(\mathbb{R}^{k}\right)^{\underline{n}}$ up to translation and scaling from its images under $\pi_{i j}$ and $s_{i j k}$.

We may define a stratification of $\widetilde{C}_{n}\left[\mathbb{R}^{k}\right]$ labelled by trees in the same fashion as for $C_{n}\left[\mathbb{R}^{k}\right]$, and the strata have a more uniform description than that of $C_{T}\left(\mathbb{R}^{k}\right)$.

Corollary 4.13. $\widetilde{C}_{T}\left(\mathbb{R}^{k}\right)$ is diffeomorphic to $\left(\widetilde{C}_{\# v}\left(\mathbb{R}^{k}\right)\right)^{V(T)}$.

Proof. We cite Lemma 4.12 and check that $\operatorname{Sim}_{k}$ is acting on each $C_{T}\left(\mathbb{R}^{k}\right)$ non-trivially only on the factor of $C_{\# v_{0}}\left(\mathbb{R}^{k}\right)$, and doing so there by its standard diagonal action.

Other results for $C_{n}\left[\mathbb{R}^{k}\right]$ have similar analogues for $\widetilde{C}_{n}\left[\mathbb{R}^{k}\right]$, which we will not state in general. One of note is that its category of strata is isomorphic to $\widetilde{\Psi}_{n}$, the category of trees with a trunk. 
Definition 4.14. (1) Define $I C_{n}[M]$ as a fiber bundle over $M$ with fiber $\widetilde{C}_{n}\left[\mathbb{R}^{m}\right]$ built from $T M$ by taking the same system of charts but choosing coordinate transformations $\widetilde{C}_{n}\left[\phi_{i j}\right]$ from $\widetilde{C}_{n}\left[\mathbb{R}^{m}\right]$ to itself, where $\phi_{i j}$ are the coordinate transformations defining $T M$.

(2) Let $I C_{e}[M]$ be defined as in Definition 3.6 but with $I C_{n}[M]$ replacing $I C_{n}(M)$.

(3) Let $D_{T}[M]$ be defined through the pull-back

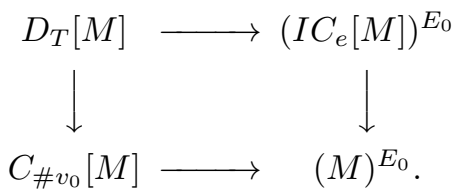

Theorem 4.15. $C_{T}[M]$ is diffeomorphic to $D_{T}[M]$.

Proof. Though by definition $C_{T}[M]$ is the closure of $C_{T}(M)$ in $C_{n}[M]$, it is also the closure of $C_{T}(M)$ in any closed subspace of $A_{n}[M]$, and we choose to consider it as a subspace of $A_{T}[M]$. The inclusion of $C_{T}(M)$ in $A_{T}[M]$ is compatible with fiber bundle structures of these spaces over $(M)^{E_{0}}$. For a general fiber bundle $F^{\prime} \rightarrow E^{\prime} \rightarrow B^{\prime}$ subspaces respectively of $F \rightarrow E \rightarrow B$, the closure $c l_{E}\left(E^{\prime}\right)$ may be defined by first extending $E^{\prime}$ to a bundle over $c_{B}\left(B^{\prime}\right)$ (which may be done locally) and then taking the closures fiber-wise. Our result follows from this general statement, the definition of $C_{\# v_{0}}[M]$ as the closure of $C_{\# v_{0}}(M)$ in $A_{\# v_{0}}[M]$, and the independence of the closure of the fibers $\widetilde{C}_{i}\left(\mathbb{R}^{m}\right)$ of $I C_{i}(M)$ in any $\widetilde{A}_{i}\left[\mathbb{R}^{k}\right]$.

4.4. Configurations in the line and associahedra. The compactification of configurations of points in the line is a fundamental case of this construction. The configuration spaces $C_{n}(\mathbb{R})$ and $C_{n}(\mathbb{I})$ are disconnected, having one component for each ordering of $n$ points. These different components each map to a different component of $A_{n}[\mathbb{R}]$, because whether $x_{i}<x_{j}$ or $x_{i}>x_{j}$ will determine a + or - for $u_{i j} \in S^{0}$. Let $C_{n}^{o}[\mathbb{R}]$ and $C_{n}^{o}[\mathbb{I}]$ denote the closure of the single component $x_{1}<\cdots<x_{n}$.

The main result of this subsection is that $\widetilde{C}_{n}[\mathbb{R}]$ is Stasheff's associahedron $A_{n-2}$, of which there is a pleasing description of $A_{n}$ due to Devadoss. The truncation of a polyhedron at some face (of any codimension) is the polyhedral subspace of points which are of a distance greater than some sufficiently small epsilon from that face. We may define $A_{n}$ as a truncation of $\Delta^{n}$. In the standard way, label the codimension one faces of $\Delta^{n}$ with elements of $\underline{n+1}$. Call $S \subset \underline{n+1}$ consecutive if $i, j \in S$ and $i<k<j$ implies $k \in S$, and call a face of $\Delta^{n}$ consecutive if the labels of codimension one faces containing it are consecutive. To obtain $A_{n}$, truncate the consecutive faces of $\Delta^{n}$, starting with the vertices, then the edges, and so forth.

Figure 4.16. The third associahedron.

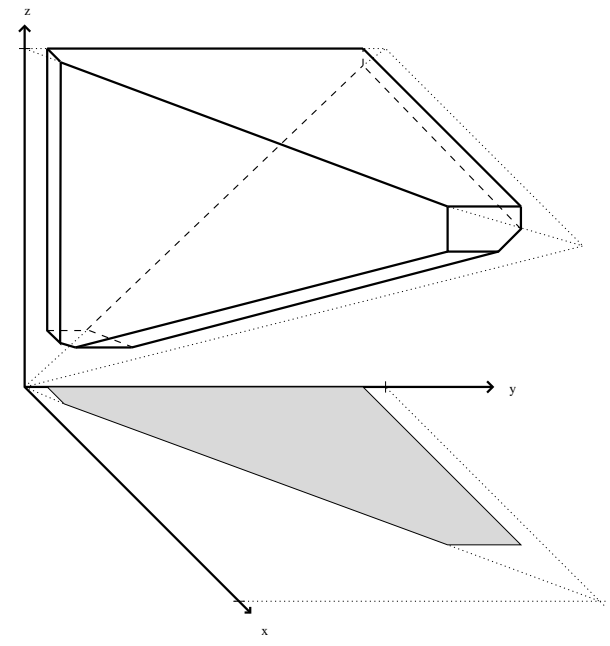


We will use a more conventional definition of the associahedron below. Closely related to the associahedron is the following sub-category of $\Psi_{n}$, whose minimal objects correspond to ways in which one can associate a product of $n$ factors in a given order.

Definition 4.17. Let $\Psi_{n}^{o}$ denote the full sub-category of $\Psi_{n}$ whose objects are $f$-trees such that the set of leaves over any vertex is consecutive and such that the root vertex has valence greater than one.

Note that any element of $\Psi_{n}^{o}$ has an embedding in the upper half plane with the root at 0 , in which the leaves occur in order and which is unique up to isotopy. We may then drop the labels from such an embedding.

In applications to knot theory, we consider manifolds with boundary which have two distinguished points in its boundary, the interval II being a fundamental case.

Definition 4.18. Given such a manifold $M$ with $y_{0}$ and $y_{1}$ in $\partial M$, let $C_{n}[M, \partial]$ be the subpsace of $C_{n+2}[M]$ which is the preimage under $p$ of points of the form $\left(y_{0}, x_{1}, \ldots, x_{n}, y_{1}\right) \in(M)^{n+2}$.

Theorem 4.19. Stasheff's associahedron $A_{n}, \widetilde{C}_{n+2}^{o}[\mathbb{R}]$ and $C_{n}^{o}[\mathbb{I}, \partial]$ are all diffeomorphic as manifolds with corners. Moreover, their barycentric subdivisions are diffeomorphic to the realization (or order complex) of the poset $\Psi_{n}^{o}$.

Proof. It is simple to check that $\widetilde{C}_{n+2}^{o}[\mathbb{R}]$ and $C_{n}^{o}[\mathbb{I}, \partial]$ are diffeomorphic using Lemma 4.12 and the fact that up to translation and scaling any $x_{0}<x_{1}<\cdots<x_{n+1} \subset \mathbb{R}$ has $x_{0}=0$ and $x_{n+1}=1$.

Next, we analyze $\widetilde{C}_{n+2}^{o}[\mathbb{R}]$ inductively using Corollary 4.13 and Theorem 4.15 First note that because the $x_{i}$ are ordered and $x_{0}$ can never equal $x_{n+1}$, the category of strata of $\widetilde{C}_{n+2}^{o}[\mathbb{R}]$ is $\Psi_{n+2}^{o}$. For $n+2=3$, $\widetilde{C}_{3}^{o}[\mathbb{R}]$ is a one-manifold whose interior is the open interval $\widetilde{C}_{3}^{o}(\mathbb{R})$ and which according to $\Psi_{3}^{o}$ has two distinct boundary points, and thus must be an interval. For $n+2=4$, the stratification according to $\Psi_{4}^{o}$ and Theorem 4.15 dictate that there are five codimension one boundary strata each isomorphic to $\widetilde{C}_{3}^{o}[\mathbb{R}]$, which we know inductively to be $\mathbb{I}$, and five vertices, each being the boundary of exactly two faces, attached smoothly (with corners) to an open two-disk, making a pentagon.

In general, $\widetilde{C}_{n+2}^{o}[\mathbb{R}]$ has an open $n$-ball for an interior and faces $\widetilde{C}_{T}^{o}[\mathbb{R}]$ which inductively we identify as $\left(A_{\# v-2}\right)^{v \in V(T)}$ glued according to the poset structure of $\Psi_{n+2}^{o}$ to make a boundary sphere, coinciding with a standard definition of $A_{n}$ using trees [23].

The last statement of the theorem follows from the general fact that if $P$ is a polytope each of whose faces (including itself) is homeomorphic to a disk, then the realization of the category of strata of $P$ is diffeomorphic to its barycentric subdivision.

In further work [18] we plan to show that the spaces $\widetilde{C}_{n}\left[\mathbb{R}^{k}\right]$ form an operad. This construction unifies the associahedra and little disks operads, and was first noticed in [12].

To review some of the salient features of the structure of $C_{n}[M]$ in general, it is helpful to think explictly about coordinates on $C_{2}^{o}[I, \partial]$. On its interior, suitable coordinates are $0<x<y<1$. Three of the faces are standard, corresponding to those for $\Delta^{2}$. They are naturally labelled $x=0, y=1$ and $x=y$, and for example we may use $y$ as a coordinate on the $x=0$ face, extending the coordinates on the interior. The final two faces are naturally labelled $0=x=y$ and $x=y=1$. Coordinates on these faces which extend interior coordinates would be $\frac{x}{y}$ and $\frac{1-y}{1-x}$, respectively. 
Figure 4.20. The second associahedron, labelled by $\Psi_{4}^{0}$, with labellings by associativity and coordinates also indicated.

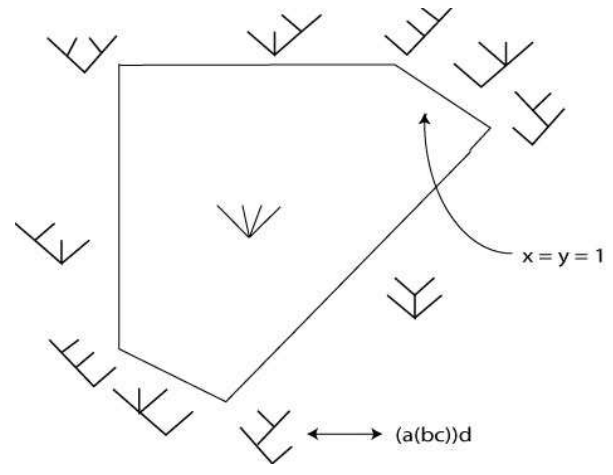

5. THE SIMPLICIAL COMPACTIFICATION

Recall Definition 1.3 of $C_{n}\langle[M]\rangle$, which we call the simplicial compactification. For $M=\mathbb{I}$, we see that $C_{n}^{o}\langle[I]\rangle$ is the closure of $C_{n}^{o}(\mathbb{I})$ in $\mathbb{I} \underline{n}$, which is simply $\Delta^{n}$. For general manifolds, we will see that $C_{n}\langle[M]\rangle$ is in some sense more complicated than $C_{n}[M]$.

Because the projection $P_{A}$ from $A_{n}[M]$ onto $A_{n}\langle[M]\rangle$ commutes with the inclusions of $C_{n}(M)$, Lemma 1.5 says that $P_{A}$ sends $C_{n}[M]$ onto $C_{n}\langle[M]\rangle$ when $M$ is compact, as we assume throughout this section.

Definition 5.1. Let $Q_{n}: C_{n}[M] \rightarrow C_{n}\langle[M]\rangle$ be the restriction of $P_{A}$.

The aim of this section is to understand $Q_{n}$, and thus the topology of $C_{n}\langle[M]\rangle$, in particular showing that this projection map is a homotopy equivalence. An immediate consequence of the surjectivity of $Q_{n}$ and Theorem 4.1 is the following.

Theorem 5.2. $C_{n}\left\langle\left[\mathbb{R}^{k}\right]\right\rangle$ is the subspace of $A_{n}\left\langle\left[\mathbb{R}^{k}\right]\right\rangle$ of points $\left(x_{i}\right) \times\left(u_{i j}\right)$ such that

(1) If $x_{i} \neq x_{k}$ then $u_{i j}=\frac{x_{i}-x_{k}}{\left\|x_{i}-x_{k}\right\|}$.

(2) $u_{i j}=-u_{j i}$, and $u_{i j}, u_{i k}$ and $u_{j k}$ are linearly dependent with $u_{i j}$ between $u_{k j}$ and $u_{i k}$ on the geodesic between them in $S^{k-1}$.

Moreover, $C_{n}\langle[M]\rangle$ is the subspace of $C_{n}\left\langle\left[\mathbb{R}^{k}\right]\right\rangle$ where all $x_{i} \in M$ and if $x_{i}=x_{j}$ then $u_{i j}$ is tangent to $M$ at $x_{i}$.

From the analysis of Lemma 3.17 we know that $\left(\pi_{i j}\right): \widetilde{C}_{n}\left(\mathbb{R}^{k}\right) \rightarrow\left(S^{k-1}\right)^{n(n-1)}$ is not injective for configurations in which all points lie on a line. These collinear configurations account for all of the difference between $C_{n}[M]$ and $C_{n}\langle[M]\rangle$.

Lemma 5.3. The map $Q_{n}$ is one-to-one except at points with some $x_{i_{1}}=\cdots=x_{i_{m}}$ and $u_{i_{h} i_{j}}= \pm u_{i_{k} i_{\ell}}$ for any $h, j, k, \ell$. The preimages of such points are diffeomorphic to a product of $A_{m-2}$ 's.

Proof. Conditions 1 and 2 of Theorem 4.1 say that in cases except these, the coordinates $d_{i j k}$ in $C_{n}[M]$ will be determined by the $x_{i}$ or $u_{i j}$ coordinates. In these cases, the $d_{i j k}$ are restricted in precisely the same manner as for the definition of $\widetilde{C}_{m}^{o}(\mathbb{R})$, which is diffeomorphic to $A_{m-2}$ by Theorem 4.19]

Thus, the preimage of any point under $Q_{n}$ will be contractible, pointing to the fact that $Q_{n}$ is a homotopy equivalence. A small difficulty is that under $Q_{n}$ points in the boundary of $C_{T}[M]$ will be identified with points in its interior. Moreover, there are identifications made which lie only in the boundary of $C_{T}[M]$. We will first treat configurations in $\mathbb{R}^{k}$ up to the action of $\operatorname{Sim}_{k}$, the building blocks for the strata of $C_{n}[M]$.

Definition 5.4. Let $\widetilde{C}_{n}\left\langle\left[\mathbb{R}^{k}\right]\right\rangle$ be the closure of the image of $\widetilde{C}_{n}\left(\mathbb{R}^{k}\right)$ under $\left(\widetilde{\pi}_{i j}\right)$ to $\widetilde{A}_{n}\left\langle\left[\mathbb{R}^{k}\right]\right\rangle=\left(S^{k-1}\right)^{C_{2}(\underline{n})}$. 
The analogue of Lemma4.12 does not hold in this setting, since as noted before $\left(\widetilde{\pi}_{i j}\right)$ is not injective for collinear configurations. Nonetheless, we will see that $\widetilde{Q}_{n}: \widetilde{C}_{n}\left[\mathbb{R}^{k}\right] \rightarrow \widetilde{C}_{n}\left\langle\left[\mathbb{R}^{k}\right]\right\rangle$ is a homotopy equivalence by exhibiting $\widetilde{Q}_{n}$ as a push-out by an equivalence. We first state some generalities about fat wedges and pushouts.

Definition 5.5. Let $\left\{A_{i} \subseteq X_{i}\right\}$ be a collection of subpsaces indexed by $i$ in some finite $\mathcal{I}$. Define the fat wedge of $\left\{X_{i}\right\}$ at $\left\{A_{i}\right\}$, denoted $\nabla_{A_{i}}^{\mathcal{I}} X_{i}$ or just $\otimes_{A_{i}} X_{i}$, to be the subspace of $\left(x_{i}\right) \in\left(X_{i}\right)^{\mathcal{I}}$ with at least one $x_{i}$ in $A_{i}$.

Suppose for each $i$ we have a map $q_{i}: A_{i} \rightarrow B_{i}$ and let $Y_{i}$ be defined by the following push-out square

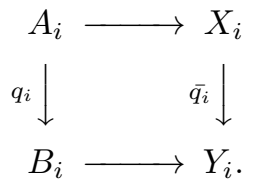

There is a map which we call $\otimes q_{i}$ from $\otimes_{A_{i}} X_{i}$ to $\otimes_{B_{i}} Y_{i}$.

Lemma 5.6. With notation as above, if each $A_{i} \hookrightarrow X_{i}$ is a (Hurewicz) cofibration and each $q_{i}$ is a homotopy equivalence then $\nabla q_{i}$ is a homotopy equivalence.

Proof. First note that in a left proper model category, if you have a diagram

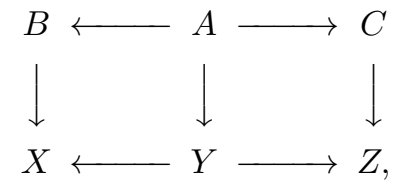

where the vertical maps are equivalences and at least one map on each of the horizontal levels is a cofibration, then the induced map of pushouts is an equivalence (see Theorem 13.5.4 in [13]). The Hurewicz model category is left proper because every space is cofibrant (see Theorem 13.1.3 in [13]).

We prove this lemma by induction. Let $\mathcal{I}=\{1, \cdots, n\}$. Inductively define the diagram $D_{j}$ as

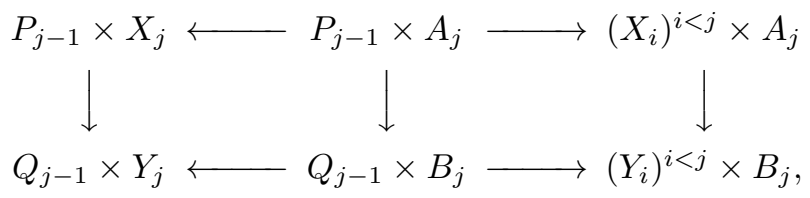

where $P_{j-1}$ is the pushout of the top row of $D_{j-1}$ and $Q_{j-1}$ is the pushout of its bottom row. Thus, $P_{n}=\otimes_{A_{i}} X_{i}$ and $Q_{n}=\otimes_{B_{i}} Y_{i}$. The verticle maps of $D_{j}$ are homotopy equivalences by induction, which is immediate if $n=1$. The left most horizontal maps are cofibrations because the product of a Hurewicz cofibration with the identity map is a Hurewicz cofibration (see Corollary 1 in [14]). We apply the pushout result above to get that $P_{n} \rightarrow Q_{n}$ is an equivalence.

For analysis of $\widetilde{Q}_{n}$, recall that for $k=1, \widetilde{\pi}_{i j}$ sends $\widetilde{C}_{n}[\mathbb{R}]$ to $S^{0}= \pm 1$.

Definition 5.7. - Let $\lambda_{m}$ be the image of the map from $\left(S^{k-1}\right) \times \widetilde{C}_{m}[\mathbb{R}] \rightarrow \widetilde{C}_{m}\left[\mathbb{R}^{k}\right]$ which sends $v, x$ to $\left(\pi_{i j}(x) \cdot v\right) \times\left(s_{i j k}(x)\right) \in \widetilde{A}_{m}\left[\mathbb{R}^{k}\right]$. We call $\lambda_{m}$ the subspace of collinear points in $\widetilde{C}_{m}\left[\mathbb{R}^{k}\right]$.

- Let $q_{m}$ denote the projection of $\left(S^{k-1}\right) \times \widetilde{C}_{m}[\mathbb{R}]$ onto $S^{k-1} \times \Sigma_{m}$ by sending each component of $\widetilde{C}_{m}[\mathbb{R}]$ to a point. Let $R_{m}$ be defined as the pushout

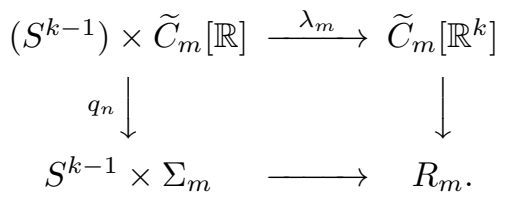


Note that $R_{m}$ maps to $\widetilde{C}_{m}\left\langle\left[\mathbb{R}^{k}\right]\right\rangle$, factoring $\widetilde{Q}_{n}$. We will see that this map is a homeomorphism on the image of $\widetilde{C}_{m}\left(\mathbb{R}^{k}\right)$ in $R_{m}$, but not on its boundary strata.

Definition 5.8. $\quad$ - By the analogue of Theorem 4.15$] \widetilde{C}_{T}\left[\mathbb{R}^{k}\right]$ is diffeomorphic to $\left(\widetilde{C_{\# v}}\left[\mathbb{R}^{k}\right]\right)^{V(T)}$. Let $\lambda_{T} \subset C_{T}\left[\mathbb{R}^{k}\right]$ be the fat wedge $\bigotimes_{\lambda_{\# v}} \widetilde{C_{\# v}}\left[\mathbb{R}^{k}\right]$.

- Let $L_{T}$ denote the fat wedge $\otimes_{S^{k-1} \times \Sigma_{\# v}} R_{\# v}$ and let $q_{T}=\bigotimes q_{\# v}: \lambda_{T} \rightarrow L_{T}$.

- Let $\bigcup_{T} \lambda_{T}$ denote the union of the $\lambda_{T}$ in $\widetilde{C}_{n}\left[\mathbb{R}^{k}\right]$. Let $\bigcup_{T} L_{T}$ denote the union of $L_{T}$ with identifications $q_{T}(x) \sim q_{T^{\prime}}(y)$ if $x \in \lambda_{T}$ is equal to $y \in \lambda_{T^{\prime}}$. Let $\bigcup_{T} q_{T}: \bigcup_{T} \lambda_{T} \rightarrow \bigcup_{T} L_{T}$ denote the projection defined compatibly by the $q_{T}$.

Theorem 5.9. The projection map $\widetilde{Q}_{n}: \widetilde{C}_{n}\left[\mathbb{R}^{k}\right] \rightarrow \widetilde{C}_{n}\left\langle\left[\mathbb{R}^{k}\right]\right\rangle$ sits in a pushout square

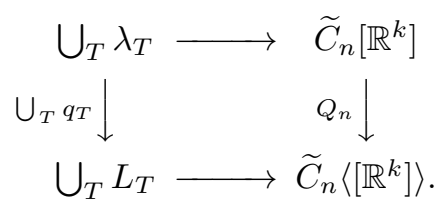

Before proving this theorem we deduce from it one of the main results of this section.

Corollary 5.10. $\widetilde{Q}_{n}$ is a homotopy equivalence.

Proof. If we apply Lemma [5.6 to the push-out squares of Equation 2 which define the $R_{\# v}$, we deduce that $q_{T}$ is a homotopy equivalence. Because of the identifications in $\bigcup_{T} L_{T}$ are essentially defined through $\bigcup_{T} q_{T}$, we deduce that $\bigcup_{T} q_{T}$ is a homotopy equivalence. Because the inclusion $\bigcup_{T} \lambda_{T} \rightarrow \widetilde{C}_{n}\left[\mathbb{R}^{k}\right]$ is a cofibration, we see that $\widetilde{Q}_{n}$ is a pushout of a homotopy equivalence through a cofibration, and thus is a homotopy equivalence itself.

Proof of Theorem 5.9. Let $X$ denote the pushout of the first three spaces in the square of Equation 3 . First note that the composite $\widetilde{Q}_{n} \circ\left(\bigcup_{T} q_{T}\right)^{-1}: \bigcup_{T} L_{T} \rightarrow \widetilde{C}_{n}\left\langle\left[\mathbb{R}^{k}\right]\right\rangle$ is well-defined, since choices of $\left(\bigcup_{T} q_{T}\right)^{-1}$ only differ in their $d_{i j k}$ coordinates. By the definition of push-out, $X$ maps to $\widetilde{C}_{n}\left\langle\left[\mathbb{R}^{k}\right]\right\rangle$ compatibly with $\widetilde{Q}_{n}$. We show that this map $F$ is a homeomorphism.

First, $F$ is onto because $\widetilde{Q}_{n}$ is onto. The key is that by construction $F$ is one-to-one. Away from $\bigcup_{T} \lambda_{T}$, $\widetilde{Q}_{n}$ is one-to-one essentially by Lemma [5.3] The projection $\widetilde{Q}_{n}$ is not one-to-one only on $x \in \widetilde{C}_{m}\left[\mathbb{R}^{k}\right]$ with some collections of $\left\{i_{j}\right\}$ such that $u_{i_{h} i_{j}}= \pm u_{i_{\ell} i_{m}}$. But such an $x$ is in $\lambda_{T(x)}$. The map $\widetilde{Q}_{n} \circ\left(\bigcup_{T} q_{T}\right)^{-1}$ is one-to-one since distinct points in $\bigcup_{T} L_{T}$ will have distinct $u_{i j}$ coordinates when lifted to $\bigcup_{T} \lambda_{T}$ which remain distinct in $\widetilde{C}_{n}\left\langle\left[\mathbb{R}^{k}\right]\right\rangle$.

Finally since it is a push-out of compact spaces, $X$ is compact. All spaces in question are subspaces of metric spaces. Thus, since $F$ is a one-to-one map between metrizable spaces whose domain is compact, it is a homeomorphism onto its image, which is all of $\widetilde{C}_{n}\left\langle\left[\mathbb{R}^{k}\right]\right\rangle$.

Theorem 5.11. The map $Q_{n}: C_{n}[M] \rightarrow C_{n}\langle[M]\rangle$ is a homotopy equivalence.

Proof. On the interior $C_{n}(M), Q_{n}$ is a homeomorphism.

The effect of $Q_{n}$ on $C_{T}[M]$ for non-trivial $T$ is through restriction to $P_{\# v_{0}}$ on the base $C_{\# v_{0}}[M]$. Working fiberwise, we see $Q_{n}$ takes each fiber bundle $\widetilde{C}_{i}\left[\mathbb{R}^{m}\right] \rightarrow I C_{i}[M] \rightarrow M$ and pushes out fiberwise to get $\widetilde{C}_{i}\left\langle\left[\mathbb{R}^{m}\right]\right\rangle \rightarrow I C_{i}\langle[M]\rangle \rightarrow M$. As $\# v_{0}<n$, by induction and Theorem [5.9] $Q_{n}$ restricted to any $C_{T}[M]$ is a homotopy equivalence. Since the inlcusions of $C_{T}[M]$ in each other are cofibrations, we can build a homotopy inverse inductively and deduce that $Q_{n}$ is a homotopy equivalence.

Unfortunately, $C_{n}\langle[M]\rangle$ is not a manifold with corners. It is however stratified by manifolds. For example, $\widetilde{C}_{n}\left\langle\left[\mathbb{R}^{k}\right]\right\rangle$ is the union of $\bigcup_{T} L_{T}$, a union of manifolds, and the complement of $\bigcup_{T} \lambda_{T}$ which is a 
submanifold of $\widetilde{C}_{n}\left[\mathbb{R}^{k}\right]$. The singularity which arises is akin to that which occurs when say a diameter of a disk gets identified to a point. We will not pursue the matter further here.

\section{Diagonal AND PROJection maps}

As we have seen, the compactifications $C_{n}[M]$ and $C_{n}\langle[M]\rangle$ are functorial with respect to embeddings of $M$. In this section we deal with projection and diagonal maps, leading to functorality with respect to $n$, viewed as the set $\underline{n}$.

Our goal is to construct maps for $C_{\# S}[M]$ and $C_{\# S}\langle[M]\rangle$ which lift the canonical maps on $M^{S}$. We start with the straightforward case of projection maps. If $\sigma: \underline{m} \rightarrow \underline{n}$ is an inclusion of sets, recall Definition 3.24 that $p_{\sigma}^{M}$ is the projection onto coordinates in the image of $\sigma$.

Proposition 6.1. Let $\sigma: \underline{m} \rightarrow \underline{n}$ be an inclusion of finite sets. There are projections $C_{\sigma}$ from $C_{n}[M]$ onto $C_{m}[M]$ and from $C_{n}\langle[M]\rangle$ onto $C_{m}\langle[M]\rangle$ which commute with each other, with $p_{\sigma}^{M}$, and its restriction to $C_{n}(M)$.

Proof. The inclusion $\sigma$ gives rise to maps from $C_{i}(\sigma): C_{i}(\underline{m}) \rightarrow C_{i}(\underline{n})$. We project $A_{n}[M]$ onto $A_{m}[M]$ through $P_{\sigma}=p_{\sigma}^{M} \times p_{C_{2}(\sigma)}^{S^{k-1}} \times p_{C_{3} \sigma}^{\mathbb{I}}$.

Because $P \circ \alpha_{n}=\alpha_{m}$ and all spaces in question are compact we apply Lemma 1.5 to see that $P_{\sigma}$ sends $C_{n}[M]$ onto $C_{m}[M]$, extending the projection from $C_{n}(M)$ to $C_{m}(M)$. By construction, $P_{\sigma}$ commutes with with $p_{\sigma}^{M}$, which is its first factor.

The projection for $C_{n}\langle[M]\rangle$ is entirely analogous, defined as the restriction of the map $P_{\sigma}^{\prime}=p_{\sigma}^{M} \times p_{C_{2}(\sigma)}^{S^{k-1}}$ : $A_{n}\langle[M]\rangle \rightarrow A_{m}\langle[M]\rangle$. We leave the routine verification that $P^{\prime}$ commutes with all maps in the statement of the theorem to the reader.

An inclusion $\sigma: \underline{m} \rightarrow \underline{n}$ gives rise to a functor $E x_{\sigma}: E x(\underline{n}) \rightarrow E x(\underline{m})$ by throwing out any exclusions involving indices not in the image of $\sigma$. The corresponding "pruning" functor for trees, $\Psi_{\sigma}: \Psi_{\underline{n}} \rightarrow \Psi_{\underline{m}}$, is defined by removing leaf vertices and edges whose label is not in the image of $\sigma$, replacing any non-root bivalent vertex along with its two edges with a single edge, and removing any vertices and edges which have all of the leaves above them removed.

Proposition 6.2. $C_{\sigma}$ sends $C_{T}[M]$ to $C_{\Psi_{\sigma}(T)}[M]$.

Proof. The effect of $C_{\sigma}$ is to omit indices not in the image of $\sigma$, so its effect on exclusion relations is precisely $E x_{\sigma}$. There is a univalent root vertex for the tree associated to $C_{\sigma}\left(C_{T}[M]\right)$ if and only if all indices $j$ for which $x_{j} \neq x_{i}$ have been omitted, which happens precisely when all leaves in $T$ except for those over a single root edge have been pruned.

If $\sigma: \underline{m} \rightarrow \underline{n}$ is not injective, it is more problematic to construct a corresponding map $C_{n}[M] \rightarrow C_{m}[M]$. Indeed, $p_{\sigma}: M^{\underline{n}} \rightarrow M^{\underline{m}}$ will not send $C_{n}(M)$ to $C_{m}(M)$, since the image of $p_{\sigma}$ will be some diagonal subspace of $M \underline{m}$ and the diagonal subspaces are precisely what are removed in defining $C_{n}(M)$. One can attempt to define diagonal maps by "doubling" points, that is adding a point to a configuration which is very close to one of the points in the configuration, but such constructions are non-canonical and will never satisfy identities which diagonal maps and projections together usually do. But, the doubling idea carries through remarkably well for compactified configuration spaces where one can "double infinitesimally". From the viewpoint of applications in algebraic topology, where projection and diagonal maps are used frequently, the diagonal maps for compactifications of configuration spaces should be of great utility.

Reflecting on the idea of doubling a point in a configuration, we see that doing so entails choosing a direction, or a unit tangent vector, at that point. Thus we first incorporate tangent vectors in our constructions. Recall that we use $S T M$ to denote the unit tangent bundle (that is, the sphere bundle to the tangent bundle) of $M$. 
Definition 6.3. If $X_{n}(M)$ is a space with a canonical map to $M^{\underline{n}}$, define $X_{n}^{\prime}(M)$ as a pull-back as follows

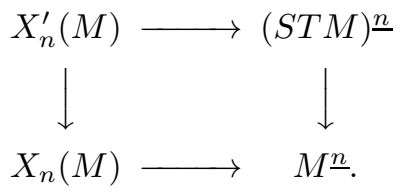

If $f_{n}: X_{n}(M) \rightarrow Y_{n}(M)$ is a map over $M^{\underline{n}}$, let $f_{n}^{\prime}: X_{n}^{\prime}(M) \rightarrow Y_{n}^{\prime}(M)$ be the induced map on pull-backs.

Lemma 6.4. $C_{n}^{\prime}[M]$ is the closure of the image of $\alpha_{n}^{\prime}: C_{n}^{\prime}(M) \rightarrow A_{n}^{\prime}[M]$. Similarly, $C_{n}^{\prime}\langle[M]\rangle$ is the closure of the image of $\beta_{n}^{\prime}$.

Proof. We check that $\operatorname{cl}_{A_{n}^{\prime}[M]}\left(\alpha_{n}^{\prime}\left(C_{n}^{\prime}(M)\right)\right)$ satisfies the definition of $C_{n}^{\prime}[M]$ as a pull-back by applying Lemma 1.5 with $\pi$ being the projection from $A_{n}^{\prime}[M]$ to $A_{n}[M]$ and $A$ being the subspace $\alpha_{n}\left(C_{n}^{\prime}(M)\right)$. The proof for $C_{n}^{\prime}\langle[M]\rangle$ proceeds similarly.

We may now treat both diagonal and projection maps for $C_{n}^{\prime}\langle[M]\rangle$. Starting with $M=\mathbb{R}^{k}$, note that $A_{n}^{\prime}\left\langle\left[\mathbb{R}^{k}\right]\right\rangle=\left(\mathbb{R}^{k} \times S^{k-1}\right)^{\underline{n}} \times\left(S^{k-1}\right)^{C_{2}(\underline{n})}$, which is canonically diffeomorphic to $\left(\mathbb{R}^{k}\right)^{\underline{n}} \times\left(S^{k-1}\right)^{\underline{n}}{ }^{2}$, as we let $u_{i i}$ be the unit tangent vector associated to the $i$ th factor of $\mathbb{R}^{k}$.

Definition 6.5. Using the product decomposition above and considering $M$ as a submanifold of $\mathbb{R}^{k}$, define $A_{\sigma}: A_{n}^{\prime}\left\langle\left[\mathbb{R}^{k}\right]\right\rangle \rightarrow A_{m}^{\prime}\left\langle\left[\mathbb{R}^{k}\right]\right\rangle$ as $p_{\sigma}^{\mathbb{R}^{k}} \times p_{\sigma^{2}}^{S^{k-1}}$ and let $F_{\sigma}$ be the restriction of $A_{\sigma}$ to $C_{n}\langle[M]\rangle$.

Proposition 6.6. Given $\sigma: \underline{m} \rightarrow \underline{n}$ the induced map $F_{\sigma}$ sends $C_{n}^{\prime}\langle[M]\rangle$ to $C_{m}^{\prime}\langle[M]\rangle$ and commutes with $p_{\sigma}^{S T M}$.

Proof. To see that the image of $F_{\sigma}$ lies in $C_{m}^{\prime}\langle[M]\rangle$, it suffices to perform the routine check that its projection to $A_{m}\langle[M]\rangle$ satisfies the conditions of Theorem [5.2] using the fact that the domain of $F_{\sigma}$, namely $C_{n}^{\prime}\langle[M]\rangle$, satisfies similar conditions. Let $\left(x_{i}\right) \times\left(u_{i j}\right)$ be $F_{\sigma}\left(\left(y_{\ell}\right) \times\left(v_{\ell m}\right)\right)$ so that $x_{i}=y_{\sigma(i)}$ and $u_{i j}=v_{\sigma(i) \sigma(j)}$.

Looking at the first condition of Theorem 5.2 $x_{i} \neq x_{j}$ means $y_{\sigma(i)} \neq y_{\sigma(j)}$. By Theorem 5.2 applied to $C_{n}\langle[M]\rangle$ we have that $v_{\sigma(i) \sigma(j)}$ is the unit vector from $y_{\sigma(i)}$ to $y_{\sigma(j)}$, which implies the corresponding fact for $u_{i j}$. Checking that $u_{i j}=-u_{j i}$ for $i \neq j$ is also immediate in this way, as is checking the linear dependence condition on $u_{i j}, u_{i k}$ and $u_{j k}$ if $i, j$ and $k$ are distinct. If $i, j$ and $k$ are not distinct, dependence is even easier to check, since two of these vectors will be equal up to sign.

We leave the rest of these routine checks to the reader.

Let $\mathcal{N}$ denote the full subcategory of the category of sets generated by the $\underline{n}$.

Corollary 6.7. Sending $\underline{n}$ to $C_{n}^{\prime}\langle[M]\rangle$ and $\sigma$ to $F_{\sigma}$ defines a contravariant functor from $\mathcal{N}$ to spaces.

Proof. We check that $F_{\sigma \circ \tau}=F_{\sigma} \circ F_{\tau}$. This follows from checking the analogous facts for $p_{\sigma}$ and $p_{\sigma^{2}}$, which are immediate.

Let $[n]=\{0, \cdots, n\}$, an ordered set given the standard ordering of integers. Recall the category $\Delta$, which has one object for each nonnegative $n$ and whose morphisms are the non-decreasing ordered set morphisms between the $[n]$. A functor from $\Delta$ to spaces is called a cosimplicial space. There is a canonical cosimplicial space often denote $\Delta^{\bullet}$ whose $n$th object is $\Delta^{n}$. To be definite we coordinatize $\Delta^{n}$ by $0=t_{0} \leq t_{1} \leq \cdots \leq t_{n} \leq t_{n+1}=1$, and label its vertices by elements of $[n]$ according to the number of $t_{i}$ equal to one. The structure maps for this standard object are the linear maps extending the maps of vertices as sets. On coordinates, the linear map corresponding to some $\sigma:[n] \rightarrow[m]$ sends $\left(t_{i}\right) \in \Delta^{n}$ to $\left(t_{\sigma^{*}(j)}\right) \in \Delta^{m}$ where $n-\sigma^{*}(j)$ is the number of $i \in[n]$ such that $\sigma(i)<m-j$.

The following corollary gives us another reason to refer to $C_{n}\langle[M]\rangle$ as the simplicial compactification of $C_{n}(M)$. For applications we are interested in a manifold $M$ equipped with one inward-pointing tangent vector $v_{0}$ and one outward-pointing unit tangent vector $v_{1}$ on its boundary. Let $C_{n}^{\prime}\langle[M, \partial]\rangle$ denote the 
subspace of $C_{n+2}^{\prime}\langle[M, \partial]\rangle$ whose first projection onto $S T M$ is $v_{0}$ and whose $n+2$ nd projection is $v_{1}$. Let $\phi: \Delta \rightarrow \mathcal{N}$ be the functor which sends $[n]$ to $\underline{n+1}$ and relabels the morphism accordingly.

Corollary 6.8. The functor which sends $[n]$ to $C_{n}^{\prime}\langle[M, \partial]\rangle$ and $\sigma:[n] \rightarrow[m]$ to the restriction of $p_{\tau}$ to $C_{n}^{\prime}\langle[M, \partial]\rangle$ where $\tau:[m+1] \rightarrow[n+1]$ is the composite $\phi \circ \sigma^{*} \circ \phi^{-1}$ defines a cosimplicial space.

This cosimplicial space models the space of knots in $M$ [22].

For $C_{n}^{\prime}[M]$, projection maps still work as in Proposition 6.1] but diagonal maps are less canonical and more involved to described. We restrict to a special class of diagonal maps for simplicity.

Definition 6.9. Let $\sigma_{i}: \underline{n+k} \rightarrow \underline{n}$ be defined by letting $K_{i}=\{i, i+1, \ldots, i+k\}$ and setting

$$
\sigma_{i}(j)= \begin{cases}j & j<K_{i} \\ i & j \in K_{i} \\ j-k & j>K_{i} .\end{cases}
$$

We must take products with associahedra in order to account for all possible diagonal maps.

Definition 6.10. - Define $\iota_{i}: I^{C_{3}(\underline{n})} \times A_{k-1} \rightarrow I^{C_{3}(\underline{n+k})}$ by recalling that $A_{k-1} \cong \widetilde{C_{k+1}(\mathbb{R}) \subset I^{C_{3}(\underline{k})}}$ and sending $\left(d_{j \ell m}\right)^{C_{3}(\underline{n})} \times\left(e_{j \ell m}\right)^{C_{3}(\underline{k})}$ to $\left(f_{j \ell m}\right)^{C_{3}(\underline{n+k})}$ with

$$
f_{j \ell m}= \begin{cases}d_{\sigma_{i}(j, \ell, m)} & \text { if at most one of } j, \ell, m \in K_{i} \\ 0 & \text { if } j, \ell \in K_{i} \text { but } m \notin K_{i} \\ 1 & \text { if } \ell, m \in K_{i} \text { but } j \notin K_{i} \\ \infty & \text { if } j, m \in K_{i} \text { but } \ell \notin K_{i} \\ e_{j-i, \ell-i, m-i} & \text { if } j, \ell, m \in K_{i} .\end{cases}
$$

- Let $D_{i, k}: A_{n}^{\prime}[M] \times A_{k-1} \rightarrow A_{n+k}^{\prime}[M]$ be the product of $A_{\sigma_{i}}: A_{n}^{\prime}\langle[M]\rangle \rightarrow A_{n+k}^{\prime}\langle[M]\rangle$ with $\iota_{i}$. Let $\delta_{k}^{i}$ denote the restriction of $D_{i}$ to $C_{n}^{\prime}[M] \times A_{k-1}$.

Proposition 6.11. $\delta_{k}^{i}$ sends $C_{n}^{\prime}[M] \times A_{k-1}$ to $C_{n+k}^{\prime}[M] \subset A_{n+k}^{\prime}[M]$.

As with Proposition [6.6] the proof is a straightforward checking that the image of $\delta_{k}^{i}$ satisfies the conditions of Theorem 4.1] One uses the fact that $C_{n}^{\prime}[M]$ satisfies those conditions, along with the definition of $\iota_{i}$. We leave closer analysis to the reader.

By analysis of the exclusion relation, we see that the image of $\delta_{k}^{i}$ lies in $C_{S}^{\prime}[M]$ where $S$ is the tree with $n+k$ leaves where leaves with labels in $K_{i}$ sit over the lone one interal vertex, which is initial for the $i$ th root edge. In general, $\delta_{k}^{i}$ sends $C_{T}^{\prime}[M]$ to $C_{T^{\prime}}^{\prime}[M]$, where $T^{\prime}$ is obtained from $T$ by adding $k+1$ leaves to $T$, each of which has the $i$ th leaf as its initial vertex.

We set $\delta^{i}=\delta_{1}^{i}: C_{n}^{\prime}[M] \rightarrow C_{n+1}^{\prime}[M]$, and note that these act as diagonal maps. One can check that composing this with the projection down back to $C_{n}^{\prime}[M]$ is the identity. Unfortunately, $\delta^{i} \delta^{i} \neq \delta^{i+1} \delta^{i}-$ see Figure 6.12 - so that the $C_{n}^{\prime}[M]$ do not form a cosimplicial space. But note that our $\delta^{2}$, when we restrict $A_{1}$ to its boundary, restricts to these two maps and thus provides a canonical homotopy between them. In fact Proposition 6.11 could be used to make an $A_{\infty}$ cosimplicial space, but it is simpler to use the $C_{n}^{\prime}\langle[M]\rangle$ if possible. 
Figure 6.12. An illustration that $\delta^{2} \delta^{2} \neq \delta^{3} \delta^{2}$.

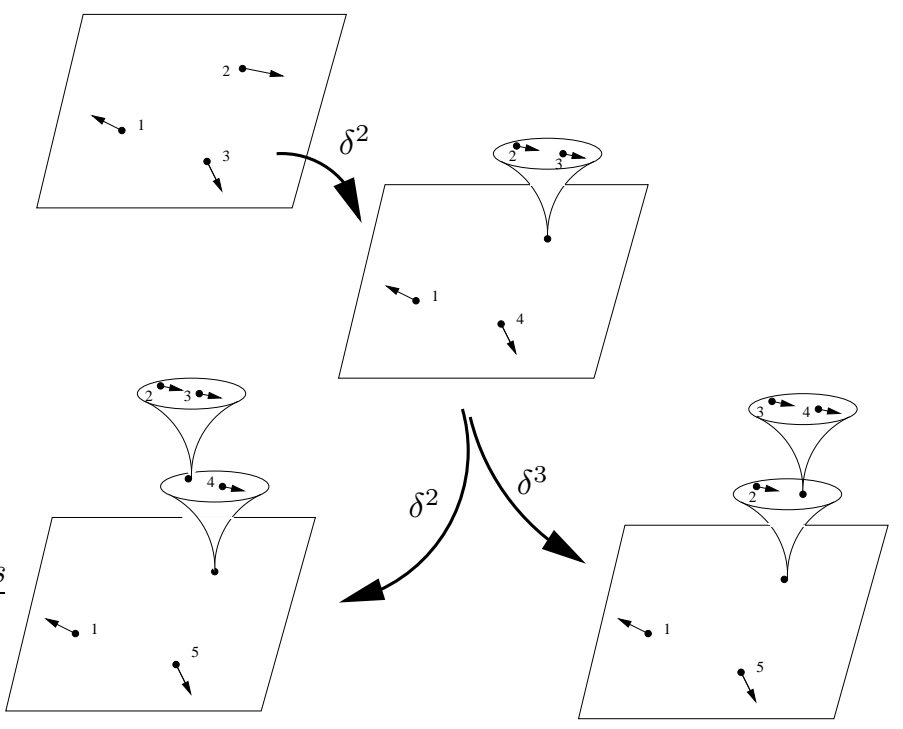

REFERENCES

[1] S. Axelrod and I. Singer. Chern-Simons perturbation theory, II. Journal of Differential Geometry 39 (1994), no. 1, 173-213.

[2] D/ Bar-Natan, S. Garoufalidis, L. Rozansky, and D. Thurston. The rhus integral of rational homology 3-spheres. I. A highly non trivial flat connection on $S^{3}$. Selecta Math. (N.S.) 8 (2002), no. 3, 315-339.

[3] R. Bott and C. Taubes. On the self-linking of knots. Topology and physics. J. Math. Phys. 35 (1994), no. 10, $5247-5287$.

[4] R. Budney, J. Conant, K. Scannell and D. Sinha. New perspectives on self-linking. Submitted, 2003.

[5] A. Cattaneo, P. Cotta-Ramusino, and R Longoni. Configuration spaces and Vassiliev classes in any dimension. Algebr. Geom. Topol. 2 (2002), 949-1000.

[6] C. De Concini and C. Procesi. Wonderful models of subspace arrangements. Selecta Mathematica (NS), 1, (1995) $459-494$.

[7] S. Devadoss. A space of cyclohedra. Discrete Comput. Geom. 29 (2003), no. 1, 61-75.

[8] E. Faddell and S. Husseini. Geometry and topology of configuration spaces. Springer 2001.

[9] E. Feitchner and D. Kozlov. Incidence combinatorics of resolutions, preprint 2003.

[10] W. Fulton and R. MacPherson. Compactification of configuration spaces. Annals of Mathematics 139 (1994), $183-225$.

[11] G. Gaiffi. Models for real subspace arrangements and stratified manifolds. Int. Math. Res. Not. (2003) no. 12, $627-656$.

[12] E. Getzler and J. Jones. Operads, homotopy algebra and iterated integrals for double loop spaces. hep-th/9403055

[13] P. Hirschhorn. Model categories and their localizations. Mathematical Surveys and Monographs, 99. American Mathematical Society, Providence, RI, 2003.

[14] J. Lillig. A union theorem for cofibrations. Arch. Math. (Basel) 24 (1973), 410-415.

[15] M. Kontsevich, Operads and motives in deformation quantization, Lett. Math. Phys. 48 (1999) 35-72.

[16] I. Kriz. On the rational homotopy type of configuration spaces. Ann. of Math. (2) 139 (1994), no. 2, $227-237$.

[17] G. Kuperberg and D. Thurston. Perturbative 3-manifold invariants by cut-and-paste topology. math.GT/9912167

[18] J. McClure, D. Sinha and J. Smith. In preparation.

[19] S. Poirier. The configuration space integral for links in $\mathbb{R}^{3}$. Algebr. Geom. Topol. 2 (2002), 1001-1050

[20] S. Saneblidze and R. Umble. A diagonal on the associahedra. math.AT/0011065 (2001).

[21] D. Sinha, Algebraic and differental topology of configuration spaces, in preparation.

[22] D. Sinha. The topology of space of knots. math.AT/0202287

[23] J. Stasheff. Homotopy associativity of $H$-spaces, I. Trans. Amer. Math. Soc. 108 (1963) $275-292$.

[24] B. Totaro. Configuration spaces of algebraic varieties. Topology 35 (1996), no. 4, 1057-1067

[25] S. Yuzvinsky. Cohomology bases for the De Concini-Procesi models of hyperplane arrangements and sums over trees. Invent. math. 127, (1997) 319-335.

Department of Mathematics, University of Oregon, Eugene, OR 97403

E-mail address: dps@math.uoregon.edu 